\title{
Conciliation through Iterated Belief Merging
}

\author{
OLIVIER GAUWIN, SÉBASTIEN KONIECZNY, PIERRE MARQUIS, \\ CRIL - CNRS, Université d'Artois, Lens, France \\ E-mail: \{gauwin, konieczny, marquis\}@cril.fr
}

\begin{abstract}
Two families of conciliation processes for intelligent agents based on an iterated merge-then-revise change function for belief profiles are introduced and studied. The processes from the first family are sceptical in the sense that at any revision step, each agent considers that her current beliefs are more important than the current beliefs of the group, while the processes from the other family are credulous. Some key features of such conciliation processes are pointed out for several merging operators; especially, the stationarity issue, the existence of consensus and the properties of the induced iterated merging operators are investigated.
\end{abstract}

Keywords: Belief merging, belief revision, conciliation.

\section{Introduction}

Belief merging is about the following question: given a set of agents whose belief bases are (typically) mutually inconsistent, how to define a belief base reflecting the beliefs of the group of agents? Formally, from a functional point of view, a belief merging operator $\triangle$ is any mapping which associates a belief base $\triangle_{\mu}(E)$ to a (non-empty) belief profile $E$ (gathering the agents' beliefs) and a belief base $\mu$ (representing some integrity constraints, which must be satisfied by the merged base). There are many different ways to address the belief merging issue in a propositional setting (see e.g. [3, 4, 20, 22-25, 28]). The variety of approaches just reflects the various ways to deal with inconsistent beliefs.

Now, the belief merging issue is not concerned with the way the resulting merged base is exploited by the group. One possibility is to suppose that all the belief bases are replaced by the (agreed) merged base. This scenario is sensible with low-level agents used for distributed computation, or in applications with distributed information sources (like distributed databases). Once the merged base has been computed, all the agents participating to the merging process share the same belief base. Such a drastic approach clearly leads to weaken the beliefs of the system. Contrastingly, when high-level intelligent agents are considered, the previous scenario looks rather unlikely: it is not reasonable to assume that the agents are ready to completely discard their current beliefs and unconditionally accept the merged base as a new belief base. It seems more adequate for them to incorporate the result of the merging process into their current belief base.

Taking such new beliefs into account calls for belief revision, which deals with the incorporation of a new piece of evidence into the beliefs of an agent [2, 15, 18]. Many belief revision operators (sometimes called 'revision schemes') can be found in the literature. From a functional point of view, a belief revision operator $*$ is any mapping which associates a belief base $K * K^{\prime}$ to a pair of belief bases $K, K^{\prime}$, where $K$ gives the initial beliefs and $K^{\prime}$ the incoming evidence.

Thus, given a revision strategy (and some revision operators-one per agent), every merging operator $\triangle$ induces what we call a conciliation operator which maps every belief

Vol. 17 No. 5, (C) The Author, 2007. Published by Oxford University Press. All rights reserved.

For Permissions, please email: journals.permissions@oxfordjournals.org

doi:10.1093/logcom/exm047 
profile $E$ (i.e. the beliefs associated to each agent at start) to a new belief profile where the new beliefs of an agent are obtained by confronting her previous beliefs with the merged base given by $E$ and $\triangle$.

In this perspective, two revision strategies can be considered. The first one consists in giving more priority to the previous beliefs; this is the strategy at work for sceptical agents. The second one, used by credulous agents, views the current beliefs of the group as more important than their own, current beliefs.

Obviously enough, it makes sense to iterate such a merge-then-revise process when the aim of agents is to reach an agreement (if possible): after a first merge-then-revise round, each agent has possibly new beliefs, defined from her previous ones and the beliefs of the group; this may easily give rise to new beliefs for the group, which must be incorporated into the previous beliefs of agents, and so on.

Let us illustrate this idea with a simple example:

\section{EXAMPLE 1}

There is a position available in some university. The committee in charge of the recruitment consists of three professors $A, B$ and $C$; the committee has to determine the right profile for the position. Three criteria are considered: managerial skills, teaching experience and research level of the candidate. Professor $A$ believes that the university does not need a teacher $(\neg t)$, and that any candidate who has a good research level $(r)$ or good managerial skills $(m)$ has the right profile. Professor $B$ believes that the university needs to recruit either a 'pure' researcher or a 'pure' teacher. Finally, Professor $C$ believes that the university needs somebody with good research level, good managerial skills and good teaching experience. Formally, the beliefs of Professors $A, B, C$ can be represented respectively by the propositional formulas $(r \vee m) \wedge \neg t, \neg m \wedge(t \leftrightarrow \neg r)$, and $r \wedge t \wedge m$. Assume now that the beliefs of the group are computed using a merging operator based on the Dalal distance and the aggregation function $\max$ (i.e. the operator $\triangle^{d_{H}}$, Max formally defined in the following). Such beliefs are equivalent to the formula $(\neg m \wedge t \wedge r) \vee(m \wedge \neg t \wedge r) \vee(m \wedge t \wedge \neg r)$. Now, each agent has to incorporate them into her own beliefs. If they all used the revision operator associated to $\triangle^{d_{H}}$, Max , i.e. Dalal revision operator [12], then the beliefs of Professor $A$ become $m \wedge \neg t \wedge r$, while the two other professors adhere to the beliefs of the group $(\neg m \wedge t \wedge r) \vee(m \wedge \neg t \wedge r) \vee(m \wedge t \wedge \neg r)$. At this stage, a consensus has been reached ${ }^{1}$ between the three professors since they now agree on $m \wedge \neg t \wedge r$, i.e. they all now believe that a good profile for the position is somebody with good managerial skills, good research level and no teaching experience. The conciliation process stops here since a further merge-then-revise round changes neither the agents beliefs nor the corresponding merged base (see Example 4 for the corresponding computations).

In this article, we define such conciliation operators, which characterize how agents' beliefs evolve according to such an iterated merge-then-revise approach. This article addresses several questions: is it possible to define conciliation operators following a mergethen-revise scheme, i.e. is there any guarantee that conciliation defined in that way is a

\footnotetext{
${ }^{1}$ While this example shows that a consensus can be reached at the end of a conciliation process, this is not always the case (see Proposition 7 and Example 4).
} 
terminating process? Do such operators always lead to a consensus between agents (i.e. agents get compatible beliefs after the conciliation)? Whenever a consensus is reached, does the conciliation process necessarily stop? Do the induced iterated merging operators satisfy rationality postulates for merging? The motivations for considering the first three questions are obvious. As to the fourth question above, the rationale is the following one: merging operators are often considered as reporting an 'average point of view' between the agents' bases; at a first glance, iterating the process might be viewed as an approach to target in a more precise way such as an 'average point of view'.

Now, defining such iterated merging operators calls for two assumptions: homogeneity (the same revision operator is used by all the agents) and compatibility (the revision operator used is the one induced by the merging operator under consideration). Without them, the 'iterated merging' operator induced by a conciliation one does not depend solely of the merging operator on which the conciliation operator is based, so it cannot be considered stricto sensu as an 'iterated merging operator'. Making those two assumptions is sensible when one deals with autonomous agents who are ready to incorporate into their beliefs the results of the merging steps, as it is the case in this article. Indeed, a possible explanation for such a behaviour is that the agents are confident in the quality of the information coming from the merging steps (especially they all agree on the merging scheme to be used, otherwise they would not participate to the conciliation process); for the scenarios where this explanation holds, the compatibility and the homogeneity assumptions are justified: it makes sense that the agents use the revision scheme associated to the merging one (so to say, the compatibility assumption justifies the homogeneity one when the merging operator is considered as 'good').

The purpose of this article is to study the two families of conciliation processes induced by the two revision strategies so as to give some answers to the questions above. We consider conciliation operators based on merging and revision operators which are rational, in the sense that they satisfy the postulates (IC0-IC8) from [22, 23] and the postulates (R1-R6) from [18], respectively (all those postulates are recalled in the following). For space reasons, we focus on conciliation processes under a uniformity assumption: all agents are either sceptical ones or credulous ones.

In a nutshell, the contribution of the article is as follows: we show that the termination of conciliation processes is guaranteed for sceptical operators, but it is only conjectured for credulous ones; we show that conciliation leads to consensus only if there is a consensus at start for sceptical operators, and does not necessarily lead to consensus for credulous operators; we also show that the conciliation process necessarily stops when a consensus is reached; finally, we show that the iterated merging operators induced by conciliation ones typically satisfy only few rationality postulates for merging; especially, when a merging operator satisfies some postulates, this does not imply that the corresponding iterated one satisfies also those postulates. Thus, iterating the merging process does not lead to 'better' merging operators.

The rest of the article is organized as follows. In the next section, some formal preliminaries are provided. Section 3 presents the main results of the article: in Section 3.1 the conciliation processes are defined, in Section 3.2 the focus is laid on the sceptical ones and in Section 3.3 on the credulous ones. In Section 4 we consider conciliation operators satisfying the compatibility and homogeneity assumptions; we investigate the connections between the conciliation processes and the merging operators they induce. Section 5 is devoted to related work. Finally, Section 6 gives some perspectives. 


\section{Conciliation through Iterated Belief Merging}

\section{Preliminaries}

We consider a propositional language $\mathcal{L}$ over a finite alphabet $\mathcal{P}$ of propositional symbols and the boolean constants $T, \perp$. An interpretation is a total function from $\mathcal{P}$ to $\{0,1\}$. The set of all the interpretations is denoted $\mathcal{W}$. An interpretation $\omega$ is a model of a formula $K$, denoted by $\omega \models K$, if it makes it true in the classical truth functional way. Let $K$ be a formula, $[K]$ denotes the set of models of $K$, i.e. $[K]=\{\omega \in \mathcal{W} \mid \omega \models K\}$. $\models$ denotes logical entailment and $\equiv$ denotes logical equivalence.

A belief base $K$ is a consistent propositional formula (or, equivalently, a finite consistent set of propositional formulas considered conjunctively), viewed up to logical equivalence.

Let $K_{1}, \ldots, K_{n}$ be $n$ belief bases (not necessarily pairwise different). We call belief profile the vector $E$ consisting of those $n$ belief bases in a specific order, $E=\left(K_{1}, \ldots, K_{n}\right)$, so that the $n$-th base gathers the beliefs of agent $n$. When $n=1$, we often write $E=K$ instead of $E=(K)$ in order to avoid heavy notations. We denote by $\wedge E$ the conjunction of the belief bases of $E$, i.e. $\bigwedge E=K_{1} \wedge \cdots \wedge K_{n}$. We say that a belief profile $E$ is consistent if $\bigwedge E$ is consistent. The union operator for belief profiles is denoted by $\sqcup$, i.e. if $E=\left(K_{1}, \ldots, K_{n}\right)$ and $E^{\prime}=\left(K_{1}^{\prime}, \ldots, K_{m}^{\prime}\right)$, then $E \sqcup E^{\prime}=\left(K_{1}, \ldots, K_{n}, K_{1}^{\prime}, \ldots, K_{m}^{\prime}\right)$.

Let $\mathcal{E}$ be the set of all finite non-empty belief profiles. Two belief profiles $E_{1}$ and $E_{2}$ from $\mathcal{E}$ are said to be equivalent (denoted by $E_{1} \equiv E_{2}$ ) if and only if there is a bijection between the profile $E_{1}$ and the profile $E_{2}$ s.t. each belief base of $E_{1}$ is logically equivalent to its image in $E_{2}$. Note that the order given by the profile is not relevant for equivalence. Two profiles $E_{1}=\left(K_{1}, \ldots, K_{n}\right)$ and $E_{2}=\left(K_{1}^{\prime}, \ldots, K_{n}^{\prime}\right)$ are said to be identical, denoted by $E_{1} \leftrightarrow E_{2}$ if and only if $\forall i \in 1 \ldots n K_{i} \equiv K_{i}^{\prime}$.

For every belief revision operator $*$, every profile $E=\left(K_{1}, \ldots, K_{n}\right)$ and every belief base $K$, we define the revision of $E$ by $K$ (resp. the revision of $K$ by $E$ ) as the belief profile given by $\left(K_{1}, \ldots, K_{n}\right) * K=\left(K_{1} * K, \ldots, K_{n} * K\right)$ (resp. $\left.K *\left(K_{1}, \ldots, K_{n}\right)=\left(K * K_{1}, \ldots, K * K_{n}\right)\right)$. Since sequences of belief profiles will be considered, we use superscripts to denote belief profiles obtained at some stage, while subscripts are used (as before) to denote belief bases within a profile. For instance, $E^{i}$ denotes the belief profile obtained after $i$ elementary evolution steps (in our framework, $i$ merge-then-revise steps), and $K_{j}^{i}$ the belief base associated to the $j$-th coordinate of vector $E^{i}$ (i.e. the beliefs of agent $j$ at step $i$ ).

\subsection{IC merging operators}

Some basic work in belief merging aims at determining sets of axiomatic properties that valuable operators should exhibit [21-24, 27, 28]. We focus here on the characterization of Integrity Constraints (IC) merging operators [22, 23].

The aim is to characterize the belief base $\triangle_{\mu}(E)$, that represents the merging of the profile $E$ under the integrity constraints $\mu . \mu$ is a formula that encodes some constraints on the result (such as physical constraints, laws, norms, etc.).

\section{Definition 1}

$\triangle$ is an IC merging operator if and only if it satisfies the following properties:

(IC0) $\triangle_{\mu}(E) \models \mu$

(IC1) If $\mu$ is consistent, then $\triangle_{\mu}(E)$ is consistent

(IC2) If $\wedge E$ is consistent with $\mu$, then $\triangle_{\mu}(E) \equiv \wedge E \wedge \mu$

(IC3) If $E_{1} \equiv E_{2}$ and $\mu_{1} \equiv \mu_{2}$, then $\triangle_{\mu_{1}}\left(E_{1}\right) \equiv \triangle_{\mu_{2}}\left(E_{2}\right)$ 
(IC4) If $K_{1} \models \mu$ and $K_{2} \models \mu$, then $\triangle_{\mu}\left(\left(K_{1}, K_{2}\right)\right) \wedge K_{1}$ is consistent if and only if $\triangle_{\mu}\left(\left(K_{1}, K_{2}\right)\right) \wedge K_{2}$ is consistent

(IC5) $\triangle_{\mu}\left(E_{1}\right) \wedge \triangle_{\mu}\left(E_{2}\right) \models \triangle_{\mu}\left(E_{1} \sqcup E_{2}\right)$

(IC6) If $\triangle_{\mu}\left(E_{1}\right) \wedge \triangle_{\mu}\left(E_{2}\right)$ is consistent, then $\triangle_{\mu}\left(E_{1} \sqcup E_{2}\right) \models \triangle_{\mu}\left(E_{1}\right) \wedge \triangle_{\mu}\left(E_{2}\right)$

(IC7) $\triangle_{\mu_{1}}(E) \wedge \mu_{2} \models \triangle_{\mu_{1} \wedge \mu_{2}}(E)$

(IC8) If $\triangle_{\mu_{1}}(E) \wedge \mu_{2}$ is consistent, then $\triangle_{\mu_{1} \wedge \mu_{2}}(E) \models \triangle_{\mu_{1}}(E)$

Two subclasses of IC merging operators have been defined. IC majority operators aim at resolving conflicts by adhering to the majority wishes, while $I C$ arbitration operators exhibit a more consensual behaviour:

DEFINITION 2

An IC majority operator is an IC merging operator which satisfies the following majority postulate:

(Maj) $\exists n \triangle_{\mu}(E_{1} \sqcup \underbrace{E_{2} \sqcup \ldots \sqcup E_{2}}_{n}) \models \triangle_{\mu}\left(E_{2}\right)$.

An IC arbitration operator is an IC merging operator which satisfies the following arbitration postulate:

(Arb)

$$
\left.\begin{array}{l}
\triangle_{\mu_{1}}\left(K_{1}\right) \equiv \triangle_{\mu_{2}}\left(K_{2}\right) \\
\triangle_{\mu_{1} \Leftrightarrow \neg \mu_{2}}\left(\left(K_{1}, K_{2}\right)\right) \equiv\left(\mu_{1} \Leftrightarrow \neg \mu_{2}\right) \\
\mu_{1} \forall \forall \mu_{2} \\
\mu_{2} \forall \mu_{1}
\end{array}\right\} \Rightarrow \triangle_{\mu_{1} \vee \mu_{2}}\left(\left(K_{1}, K_{2}\right)\right) \equiv \triangle_{\mu_{1}}\left(K_{1}\right) .
$$

See $[22,23]$ for further explanations about those two postulates and the behaviour of the corresponding merging operators.

Among IC merging operators are the distance-based ones. In order to present such operators, one first needs to recall the following notions of pseudo-distance and aggregation function:

\section{DEFINITION 3}

A pseudo-distance $d$ between interpretations is a total function $d: \mathcal{W} \times \mathcal{W} \mapsto \mathbb{R}^{+}$such that for any $\omega, \omega^{\prime} \in \mathcal{W}, d\left(\omega, \omega^{\prime}\right)=d\left(\omega^{\prime}, \omega\right)$, and $d\left(\omega, \omega^{\prime}\right)=0$ if and only if $\omega=\omega^{\prime}$.

Two widely used pseudo-distances between interpretations are Dalal distance [12], denoted by $d_{H}$, which is the Hamming distance between interpretations (i.e. the number of propositional variables on which the two interpretations differ); and the drastic distance, denoted by $d_{D}$, which is the simplest pseudo-distances one can define: it gives 0 if the two interpretations are the same one, and 1 otherwise.

\section{DEFINITION 4}

An aggregation function $f$ is a total function ${ }^{2}$ associating a non-negative real number to every finite tuple of non-negative real numbers s.t. for any $x_{1}, \ldots, x_{n}, x, y \in I R^{+}$:

- if $x \leq y$, then $f\left(x_{1}, \ldots, x, \ldots, x_{n}\right) \leq f\left(x_{1}, \ldots, y, \ldots, x_{n}\right)$.

(non-decreasingness)

- $f\left(x_{1}, \ldots, x_{n}\right)=0$ if and only if $x_{1}=\ldots=x_{n}=0$.

(minimality)

- $f(x)=x$.

(identity)

\footnotetext{
${ }^{2}$ More precisely it is a family of such functions, one for each $n \in I N^{*}$.
} 
TABLE 1. Distance-based merging operators at work

\begin{tabular}{l|llll|lrl}
\hline $\mathcal{W}$ & $K_{1}$ & $K_{2}$ & $K_{3}$ & $K_{4}$ & $d_{d_{H}, \mathrm{Max}}$ & $d_{d_{H}, \Sigma}$ & $d_{d_{H}, \mathrm{GMax}}$ \\
\hline$(0,0,0,0)$ & 3 & 3 & 0 & 2 & 3 & 8 & $(3,3,2,0)$ \\
$(0,0,0,1)$ & 3 & 3 & 1 & 3 & 3 & 10 & $(3,3,3,1)$ \\
$(0,0,1,0)$ & 2 & 2 & 1 & 1 & $\mathbf{2}$ & 6 & $(\mathbf{2}, \mathbf{2}, \mathbf{1}, \mathbf{1})$ \\
$(0,0,1,1)$ & 2 & 2 & 2 & 2 & $\mathbf{2}$ & 8 & $(2,2,2,2)$ \\
$(0,1,0,0)$ & 2 & 2 & 1 & 1 & $\mathbf{2}$ & 6 & $(\mathbf{2}, \mathbf{2}, \mathbf{1}, \mathbf{1})$ \\
$(0,1,0,1)$ & 2 & 2 & 2 & 2 & $\mathbf{2}$ & 8 & $(2,2,2,2)$ \\
$(0,1,1,0)$ & 1 & 1 & 2 & 0 & 2 & 4 & $(2,1,1,0)$ \\
$(0,1,1,1)$ & 1 & 1 & 3 & 1 & 3 & 6 & $(3,1,1,1)$ \\
$(1,0,0,0)$ & 2 & 2 & 1 & 2 & $\mathbf{2}$ & 7 & $(2,2,2,1)$ \\
$(1,0,0,1)$ & 2 & 2 & 2 & 3 & 3 & 9 & $(3,2,2,2)$ \\
$(1,0,1,0)$ & 1 & 1 & 2 & 1 & 2 & 5 & $(2,1,1,1)$ \\
$(1,0,1,1)$ & 1 & 1 & 3 & 2 & 3 & 7 & $(3,2,1,1)$ \\
$(1,1,0,0)$ & 1 & 1 & 2 & 1 & 2 & 5 & $(2,1,1,1)$ \\
$(1,1,0,1)$ & 1 & 1 & 3 & 2 & 3 & 7 & $(3,2,1,1)$ \\
$(1,1,1,0)$ & 0 & 0 & 3 & 0 & 3 & 3 & $(3,0,0,0)$ \\
$(1,1,1,1)$ & 0 & 0 & 4 & 1 & 4 & $\mathbf{5}$ & $(4,1,0,0)$ \\
\hline
\end{tabular}

Widely used functions are the max [23, 28], the sum $\Sigma[22,25,28]$, or the leximax GMax $[22,23]$. The leximax GMax orders the tuple of its arguments, by sorting them in a decreasing order (for instance, $\operatorname{GMax}(0,1,0,2,3)=(3,2,1,0,0)$ ), and by comparing them for lexicographic ordering (for instance $(3,2,1,0,0)<(3,2,2,1,0))^{3}$

Given a pseudo-distance $d$ and an aggregation function $f$, one can define a distance-based merging operator $\triangle^{d, f}$ :

\section{DEFINITION 5}

Let $d$ be a pseudo-distance between interpretations and $f$ be an aggregation function. The result $\triangle_{\mu}^{d, f}(E)$ of the merging of $E$ given the integrity constraints $\mu$ is defined semantically by:

$$
\left[\triangle_{\mu}^{d, f}(E)\right]=\min \left([\mu], \leq_{E}\right)
$$

where

- $\omega \leq_{E} \omega^{\prime}$ if and only if $d(\omega, E) \leq d\left(\omega^{\prime}, E\right)$.

- $d(\omega, E)=f_{K_{i} \in E}\left(d\left(\omega, K_{i}\right)\right)$. $^{4}$

- $d(\omega, K)=\min _{\omega^{\prime} \models K} d\left(\omega, \omega^{\prime}\right)$.

Let us illustrate the previous definitions on an example.

EXAMPLE 2

Let us consider a belief profile $E=\left(K_{1}, K_{2}, K_{3}, K_{4}\right)$ and an integrity constraint $\mu$ defined on a propositional language built over four symbols, as follows: $[\mu]=\mathcal{W} \backslash\{(0,1,1,0)$, $(1,0,1,0),(1,1,0,0),(1,1,1,0)\}, \quad\left[K_{1}\right]=\{(1,1,1,1),(1,1,1,0)\}, \quad\left[K_{2}\right]=\{(1,1,1,1),(1,1,1,0)\}$, $\left[K_{3}\right]=\{(0,0,0,0)\}$ and $\left[K_{4}\right]=\{(1,1,1,0),(0,1,1,0)\}$.

The computations are reported in Table 1. The shadowed lines correspond to the interpretations rejected by the integrity constraints. Thus the result has to be taken among the

\footnotetext{
${ }^{3}$ This definition can be scalarized to give a number, as required by Definition 4, cf. [20].

${ }^{4} d_{d, f}(\omega, E)$ is a more correct, yet heavy, notation for $d(\omega, E)$.
} 
interpretations that are not shadowed. Each $K_{i}$ column shows the Dalal distance between each interpretation and the corresponding source. The last three columns show the distance between each interpretation and the profile according to the different aggregation functions. So the selected interpretations for the corresponding operators are the ones with minimal aggregated distance.

With the $\triangle^{d_{H}}$, Max operator, the minimum distance is 2 and the chosen interpretations are $\left[\triangle_{\mu}^{d_{H}, \operatorname{Max}}(E)\right]=\{(0,0,1,0),(0,0,1,1),(0,1,0,0),(0,1,0,1),(1,0,0,0)\}$.

With the $\triangle^{d_{H}}$, GMax operator, the result is $\left[\triangle_{\mu}^{d_{H}, \operatorname{GMax}}(E)\right]=\{(0,0,1,0),(0,1,0,0)\}$.

Finally, if one chooses $\triangle^{d_{H}, \Sigma}$ for solving the conflicts according to majority wishes, the result is $\left[\triangle_{\mu}^{d_{H}, \Sigma}(E)\right]=\{(1,1,1,1)\}$.

\subsection{Merging vs. revision}

Belief revision operators deal with the incorporation of a new piece of evidence into the beliefs of an agent; typically, the new piece of evidence is considered more reliable than the beliefs of the agent $[2,15,18]$. More generally, belief revision operators can be used to aggregate two pieces of information, when one takes precedence on the other.

Let us recall the Katsuno and Mendelzon [18] postulates for belief revision operators. Let $\varphi$ and $\mu$ be two formulas from $\mathcal{L}$. The operator $*$ is a $K M$ revision operator if and only if it satisfies the following postulates:

(R1) $\varphi * \mu$ implies $\mu$

(R2) If $\varphi \wedge \mu$ is consistent then $\varphi * \mu \equiv \varphi \wedge \mu$

(R3) If $\mu$ is consistent then $\varphi * \mu$ is consistent

(R4) If $\varphi_{1} \equiv \varphi_{2}$ and $\mu_{1} \equiv \mu_{2}$ then $\varphi_{1} * \mu_{1} \equiv \varphi_{2} * \mu_{2}$

(R5) $(\varphi * \mu) \wedge \phi$ implies $\varphi *(\mu \wedge \phi)$

(R6) If $(\varphi * \mu) \wedge \phi$ is consistent then $\varphi *(\mu \wedge \phi)$ implies $(\varphi * \mu) \wedge \phi$

When a finite propositional language is considered (which is the case in this article), Katsuno and Mendelzon postulates are equivalent to AGM ones ([2, 15], see [18] for full justifications).

Interestingly, belief revision operators can be viewed as special cases of belief merging operators when applied to singleton profiles, as stated below:

Proposition 1 [23]

If $\triangle$ is an IC merging operator (it satisfies (IC0-IC8)), then the operator $* \Delta$, defined by $K * \triangle \mu=\triangle_{\mu}(K)$, is an AGM revision operator (it satisfies $(\boldsymbol{R} \mathbf{1}-\boldsymbol{R} \boldsymbol{6})$ ) [18]. This operator is called the revision operator associated to the merging operator $\triangle$.

Thanks to this proposition, to each IC merging operator one can straightforwardly associate an AGM revision operator. For instance, all the operators $\triangle^{d_{H}, f}$ (where $f$ is any aggregation function) are associated to the Dalal revision operator [12, 18].

\section{Conciliation operators}

Conciliation operators aim at reflecting the evolution of belief profiles, typically towards the achievement of some agreements between agents. Conciliation can be viewed as a simple form of negotiation, where the way beliefs may evolve is uniform and preset. 


\subsection{Definitions}

Let us first give the following, very general, definition of conciliation operators:

\section{DeFinition 6}

A conciliation operator is a function from the set of belief profiles to the set of belief profiles.

This definition does not impose any strong constraints on the result, except that each resulting belief profile is solely defined from the given one. This does not prevent conciliation operators from taking advantage of additional information as parameters. For instance, integrity constraints representing norms or laws can be taken into account. There are several ways to do it; if one assumes that the agents' beliefs must obey such laws, one can discard from the profile any agent who does not satisfy this requirement; one can also ask each agent to revise her own beliefs by the integrity constraints as a preliminary step so as to ensure it. In the following we adhere to a more liberal attitude and require integrity constraints to be satisfied at the group level, i.e. we do not ask that the beliefs of each agent satisfy the constraints. This relaxation is all the more important when conciliation is about preferences (i.e. goals): each agent is about to change her preferences in the light of the preferences of other agents, with the objective of achieving some agreements; each agent is free to have her own preferences, even if they are infeasible. Nevertheless, the most preferred alternatives at the group level have to be feasible.

Clearly, pointing out the desirable properties for such conciliation operators is an interesting issue. We leave this for future work, but one can note that the social contraction functions introduced by Booth $[10,11]$ are a step in this direction.

In the following, we focus on a particular family of conciliation operators: conciliation operators induced by an iterated merge-then-revise process. The idea is to compute a merged base from the profile, to revise the beliefs of each agent by it, and to repeat this process until a fixed point is reached. When such a fixed point exists, the conciliation operator is defined and the resulting profile is the image of the original profile by this operator. Indeed, when a fixed point has been reached, incorporating the beliefs of the group has no further impact on the own beliefs of each agent; in some sense, each agent did her best w.r.t. the group, given her revision operator. Then there are two possibilities: either a consensus has been obtained, or no consensus can be obtained that way:

\section{DeFinition 7}

There is a consensus for a belief profile $E$ if and only if $E$ is consistent (with the integrity constraints).

The existence of a consensus for a belief profile just means that the associated agents agree on at least one possible world. When this is the case, the models of the corresponding merged base w.r.t. any IC merging operator reduce to such possible worlds ((IC2) ensures it).

Interestingly, it can be shown that the existence of a consensus at some stage of the merge-then-revise process is sufficient to ensure the existence of a fixed point, hence the termination of the process. Before pointing out this result formally, let us first define the iterated merge-then-revise process in a more precise way. Indeed, focusing on classical AGM belief revision operators, there are two uniform ways to define the process, depending of the relative confidence of the agents in their own beliefs and in the result 
of the merging process. This leads to two families of operators: credulous and sceptical operators.

\section{DeFinition 8}

Let $\triangle$ be an IC merging operator, and for any $n \in I N^{*}$, let $*_{1}, \ldots, *_{n}$ be $n$ AGM revision operators. Let $E=\left(K_{1}, \ldots, K_{n}\right)$ be a belief profile and $\mu$ an integrity constraint. We define the sequence $\left(E_{c}^{i}\right)_{i \geq 0}$ (depending on $\triangle, *_{1}, \ldots, *_{n}, E$, and $\mu$ ) by:

$E_{c}^{i}=\left(K_{1}^{i}, \ldots, K_{n}^{i}\right)$, where each $K_{j}^{i}$ is defined inductively by:

- $K_{j}^{0}=K_{j}$,

- $K_{j}^{i+1}=K_{j}^{i} *_{j} \triangle_{\mu}\left(E_{c}^{i}\right)$.

The credulous Iterated Merging Conciliation (IMC) operator induced by $\triangle$ and $*_{1}, \ldots, *_{n}$ is the function that associates $E$ to $E_{c}^{k}$, where $k$ is the least integer $i$ such that $E_{c}^{i} \leftrightarrow E_{c}^{i+1}$, and is undefined otherwise. We denote by $E_{c}^{*}=E_{c}^{k}$ the resulting profile.

\section{DeFinition 9}

Let $\triangle$ be an IC merging operator, and for any $n \in I N^{*}$, let $*_{1}, \ldots, *_{n}$ be $n$ AGM revision operators. Let $E=\left(K_{1}, \ldots, K_{n}\right)$ be a belief profile and $\mu$ an integrity constraint. We define the sequence $\left(E_{s}^{i}\right)_{i>0}$ (depending on $\triangle, *_{1}, \ldots, *_{n}, E$, and $\mu$ ) by:

$E_{s}^{i}=\left(K_{1}^{i}, \ldots, K_{n}^{i}\right)$, where each $K_{j}^{i}$ is defined inductively by:

- $K_{j}^{0}=K_{j}$,

- $K_{j}^{i+1}=\triangle_{\mu}\left(E_{s}^{i}\right) *_{j} K_{j}^{i}$.

The sceptical IMC operator induced by $\triangle$ and $*_{1}, \ldots, *_{n}$ is the function that associates $E$ to $E_{s}^{k}$, where $k$ is the least integer $i$ such that $E_{s}^{i} \leftrightarrow E_{s}^{i+1}$, and is undefined otherwise. We denote by $E_{s}^{*}=E_{s}^{k}$ the resulting profile.

Every IMC operator induces a merging operator: the operator that associates to each profile the merged base of the resulting profile. We call it the IM operator associated to the IMC operator. Formally:

DEFINITION 10

Let $\triangle$ be an IC merging operator, and for any $n \in I N^{*}$, let $*_{1}, \ldots, *_{n}$ be $n$ AGM revision operators.

- The sceptical IM operator induced by $\triangle$ and $*_{1}, \ldots, *_{n}$ is the function that maps every profile $E$ and every integrity constraint $\mu$ to $\triangle_{\mu}\left(E_{s}^{*}\right)$.

- The credulous IM operator induced by $\triangle$ and $*_{1}, \ldots, *_{n}$ is the function that maps every profile $E$ and every integrity constraint $\mu$ to $\triangle_{\mu}\left(E_{c}^{*}\right)$.

An important point is that, under rationality assumptions about the merging operator and the revision operators at work in the conciliation process, once a consensus has been obtained at some stage, the process stops since the resulting profile is found.

From now on, in order to alleviate the notations, when we talk about merging operators, we mean IC merging operators, and 'revision operator' is used as a short for AGM revision operator.

Proposition 2

For any credulous (resp. sceptical) IMC operator induced by a merging operator and $n$ belief revision operators, for any profile $E$ and any integrity constraint $\mu$, if a consensus exists for $E_{c}^{i}\left(\operatorname{resp} . E_{s}^{i}\right)$, then $E_{c}^{*}=E_{c}^{i+1}\left(\operatorname{resp} . E_{s}^{*}=E_{s}^{i+1}\right)$. 
As expected, if a consensus can be reached using an iterated merging conciliation operator, all the agents share the same beliefs once the conciliation has been achieved. Such beliefs consist of the conjunction of all bases (with the constraints) from the profile for which a consensus has been found.

Let us now consider two additional properties on conciliation operators: homogeneity and compatibility.

\section{DEFINITION 11}

Let $\triangle$ be a merging operator, and, for any $n \in I^{*}$, let $*_{1}, \ldots, *_{n}$ be $n$ revision operators. An iterated merging conciliation operator is said to be:

- homogeneous if all the agents use the same revision operator: $*_{1}=\ldots=*_{n}=*$,

- compatible if the revision operator $*$ is associated to the merging operator $\triangle: *=* \Delta$.

Under the compatibility and homogeneity $(\mathrm{CH})$ assumptions, defining an IMC operator just requires to make precise the belief merging operator under use and the revision strategy (sceptical or credulous). Such an IMC operator is referred to as a CHIMC operator:

\section{DEFINITION 12}

Let $\triangle$ be a merging operator. Let $E$ be any belief profile and $\mu$ an integrity constraint. We define the sequence $\left(E_{s}^{i}\right)_{i \geq 0}$ (depending on both $\triangle, E$ and $\mu$ ) by:

- $E_{s}^{0}=E$,

- $E_{S}^{i+1}=\triangle_{\mu}\left(E_{s}^{i}\right) * E_{s}^{i}$.

The sceptical CHIMC operator $\boldsymbol{\Delta}^{*}$ induced by $\triangle$ is defined by $\boldsymbol{\Delta}_{\mu}^{*}(E)=E_{s}^{k}$, where $k$ is the least integer $i$ such that $E_{s}^{i} \leftrightarrow E_{s}^{i+1}$, and $\boldsymbol{\Delta}_{\mu}^{*}(E)$ is undefined otherwise. We denote by $E_{s}^{*}=E_{s}^{k}$ the resulting profile.

DEFINITION 13

Let $\triangle$ be a merging operator. Let $E$ be any belief profile and $\mu$ an integrity constraint. We define the sequence $\left(E_{c}^{i}\right)_{i \geq 0}$ by:

- $E_{c}^{0}=E$,

- $E_{c}^{i+1}=E_{c}^{i} * \Delta \triangle_{\mu}\left(E_{c}^{i}\right)$.

The credulous CHIMC operator ${ }^{*} \boldsymbol{\Delta}$ induced by $\triangle$ is defined by ${ }^{*} \boldsymbol{\Lambda}_{\mu}(E)=E_{c}^{k}$, where $k$ is the least integer $i$ such that $E_{c}^{i} \leftrightarrow E_{c}^{i+1}$, and ${ }^{*} \boldsymbol{\Delta}_{\mu}(E)$ is undefined otherwise. We denote by $E_{c}^{*}=E_{c}^{k}$ the resulting profile.

Every CHIMC operator induces a merging operator: the operator that associates to each profile the merged base of the resulting profile. Formally:

\section{DEFINITION 14}

Let $\triangle$ be a merging operator, and $*$ its associated revision operator.

- The sceptical CHIM operator induced by $\triangle$ is the function $\triangle^{*}$ that maps every profile $E$ and integrity constraint $\mu$ to $\triangle_{\mu}\left(E_{s}^{*}\right)$.

- The credulous CHIM operator induced by $\triangle$ is the function ${ }^{*} \triangle$ that maps every profile $E$ and integrity constraint $\mu$ to $\triangle_{\mu}\left(E_{c}^{*}\right)$.

Let us now study the key features of the two sequences $\left(E_{s}^{i}\right)_{i \geq 0}$ and $\left(E_{c}^{i}\right)_{i \geq 0}$ and the properties of the corresponding IMC operators. 


\subsection{Properties of sceptical IMC operators}

We start with sceptical IMC operators. Let us first give an important monotony property, which states that the conciliation process given by any IC merging operator and any $n$-uple of AGM revision operators may only lead to strengthen the beliefs of each agent:

Proposition 3

Let $K_{j}^{i}$ denote the belief base corresponding to agent $j$ in the belief profile $E_{s}^{i}$ characterized by the initial belief profile $E$, the integrity constraint $\mu$, the merging operator $\triangle$ and $n$ revision operators $*_{1}, \ldots, *_{n}$. For every $i, j$, we have $K_{j}^{i+1} \models K_{j}^{i}$.

Another interesting property is that the sequence of profiles and the corresponding sequence of merged bases are equivalent with respect to stationarity: ${ }^{5}$

Proposition 4

Let $E$ be a belief profile, $\triangle$ be a merging operator, and $n$ revision operators $*_{1}, \ldots, *_{n}$. Let $\mu$ be any integrity constraint. If the sequence $\left(E_{s}^{i}\right)_{i>0}$ is stationary from $i$, then the sequence $\left(\triangle_{\mu}\left(E_{s}^{i}\right)\right)_{i \geq 0}$ is stationary from $i$. Conversely, if the sequence $\left(\triangle_{\mu}\left(E_{s}^{i}\right)\right)_{i \geq 0}$ is stationary from $i$, then the sequence $\left(E_{s}^{i}\right)_{i \geq 0}$ is stationary from $i+1$.

On this ground, it is easy to prove that the sequence $\left(E_{s}^{i}\right)_{i \geq 0}$ is stationary for every profile $E$. Accordingly, the sceptical conciliation operator and the induced sceptical IM operator are defined for every $E$ :

PROPOSITION 5

For every belief profile $E$, every integrity constraint $\mu$, every merging operator $\triangle$, and every $n$-uple of revision operators $*_{1}, \ldots, *_{n}$, the stationarity of $\left(E_{s}^{i}\right)_{i \geq 0}$ is reached from an integer upper bounded by $\sum_{K \in E} \#([K])-\#(E)$. Therefore, the sceptical IMC operator induced by $\triangle$ and $*_{1}, \ldots, *_{n}$, and the associated sceptical IM operator are total functions.

Under the compatibility and homogeneity assumptions, we can prove that the number of iterations needed to reach the fixed point of $\left(E_{s}^{i}\right)_{i>0}$ is 1 , provided that the underlying merging operator is one of $\triangle^{d_{D}, \operatorname{Max}}, \triangle^{d_{D}, \Sigma}, \triangle^{d_{D}, \text { GMax }}$. This is an easy consequence of the following proposition:

PROPOSITION 6

If the merging operator $\triangle$ is $\triangle^{d_{D}, \mathrm{Max}}$ or $\triangle^{d_{D}, \Sigma}=\triangle^{d_{D} \text {, GMax }}$ then for any profile $E=\left(K_{1}, \ldots, K_{n}\right)$ and any integrity constraint $\mu$, the sceptical CHIMC operator given by $\boldsymbol{\Delta}_{\mu}^{*}(E)=E_{s}^{*}=\left(K_{1}^{*}, \ldots, K_{n}^{*}\right)$, is s.t. for every $j$ :

$$
K_{j}^{*} \equiv \begin{cases}K_{j} \wedge \triangle_{\mu}(E) & \text { if consistent } \\ K_{j} & \text { otherwise. }\end{cases}
$$

Furthermore, the resulting profile is obtained after at most one iteration (i.e. for every $i>0$, $\left.E_{s}^{i+1} \leftrightarrow E_{s}^{i}\right)$.

We have no direct (i.e. non-iterative) definition for any sceptical CHIMC operator based on an IC merging operator defined from Dalal distance. Let us give an example of such an operator:

\footnotetext{
${ }^{5}$ An (infinite) sequence $\left(u_{i}\right)_{i \geq 0}=u_{0}, \ldots u_{n}, \ldots$ is said to be stationary from $i$ if and only if $\forall j \geq i, u_{j+1}=u_{j}$. It is said to be stationary if and only if there exists an integer $i$ s.t. it is stationary from $i$.
} 
TABLE 2. The sceptical CHIMC operator $\boldsymbol{\Delta}_{\mu}^{* d_{H}}$, GMax

\begin{tabular}{l|lll|l}
\hline $\mathcal{W}$ & $K_{1}^{i}$ & $K_{2}^{i}$ & $K_{3}^{i}$ & $E_{\triangle_{\mu}\left(E^{i}\right)}^{i}$ \\
\hline$(0,0,0)$ & $0,1,1$ & $2,2,2$ & $0,1,1$ & $(2,0,0)_{1},(2,1,1)_{1},(2,1,1)_{1}$ \\
$(0,0,1)$ & $0,0,0$ & $1,1,3$ & $1,1,1$ & $(1,1,0)_{0},(1,1,0)_{0},(3,1,0)_{1}$ \\
$(0,1,0)$ & $0,0,2$ & $1,1,1$ & $1,2,2$ & $(1,1,0)_{0},(2,1,0)_{1},(2,2,1)_{2}$ \\
$(0,1,1)$ & $1,1,1$ & $0,0,2$ & $1,2,2$ & $(1,1,0)_{0},(2,1,0)_{1},(2,2,1)_{2}$ \\
$(1,0,0)$ & $1,2,2$ & $1,1,1$ & $0,0,0$ & $(1,1,0)_{0},(2,1,0)_{1},(2,1,0)_{0}$ \\
$(1,0,1)$ & $1,1,1$ & $1,2,2$ & $0,0,0$ & $(1,1,0)_{0},(2,1,0)_{1},(2,1,0)_{0}$ \\
$(1,1,0)$ & $1,1,3$ & $0,0,0$ & $1,1,1$ & $(1,1,0)_{0},(1,1,0)_{0},(3,1,0)_{1}$ \\
$(1,1,1)$ & $2,2,2$ & $0,1,1$ & $0,1,1$ & $(2,0,0)_{1},(2,1,1)_{1},(2,1,1)_{1}$ \\
\hline
\end{tabular}

\section{EXAMPLE 3}

Let us consider the profile $E=\left(K_{1}, K_{2}, K_{3}\right)$ with $\left[K_{1}\right]=\{(0,0,0),(0,0,1),(0,1,0)\}$, $\left[K_{2}\right]=\{(0,1,1),(1,1,0),(1,1,1)\}, \quad\left[K_{3}\right]=\{(0,0,0),(1,0,0),(1,0,1),(1,1,1)\}, \quad$ no integrity constraints $(\mu \equiv \mathrm{T})$, and the sceptical CHIMC operator $\boldsymbol{\Delta}^{* d_{H} \mathrm{GMax}}$ induced by $\triangle^{d_{H} \mathrm{GMax}}$. The complete process is depicted in Table 2. Each $K_{i}$ column shows the Dalal distance between each interpretation and $K_{i}$. The last column shows the distance between each interpretation and the profile according to the aggregation function. The selected interpretations for the corresponding operators are the ones with minimal aggregated distance. In the last column for sceptical operators is shown in subscript the distance used for revision (one can check that the 0s correspond to the models of the merged base).

Since there are several (three in that case) iterations, we sum up the three tables (corresponding to the three merging steps) in a single one. For example in column $K_{1}^{i}$, the first number ( 0 ) denotes the distance between the interpretation $\omega$ and $K_{1}^{1}$, the second one (1) the distance between $\omega$ and $K_{1}^{2}$, and so on.

Let us explain the full process in details. The first profile is $E^{0}=E$. The first merging iteration gives as result $\left[\triangle^{d_{H}, \operatorname{GMax}}\left(E^{0}\right)\right]=\{(0,0,1),(0,1,0),(0,1,1),(1,0,0),(1,0,1),(1,1,0)\}$. Then, every agent revises the result of the merging with its old beliefs, i.e. $K_{i}^{1}=\triangle^{d_{H}, \text { GMax }}\left(E^{0}\right) * K_{i}^{0}$, so $\left[K_{1}^{1}\right]=\{(0,0,1),(0,1,0)\},\left[K_{2}^{1}\right]=\{(0,1,1),(1,1,0)\}$ and $\left[K_{3}^{1}\right]=\{(1,0,0),(1,0,1)\}$. Since each of the three bases is consistent with the merged base, the new base of each agent is just the conjunction of her previous base with the merged base (in accordance with revision postulates). Then, the second merging iteration gives $\left[\triangle^{d_{H}, \operatorname{GMax}}\left(E^{1}\right)\right]=\{(0,0,1),(1,1,0)\}$, and the revision of each base gives $\left[K_{1}^{2}\right]=\{(0,0,1)\},\left[K_{2}^{2}\right]=\{(1,1,0)\}$, and $\left[K_{3}^{2}\right]=\{(1,0,0),(1,0,1)\}$. The third iteration step gives $\left[\triangle^{d_{H}, \operatorname{GMax}}\left(E^{2}\right)\right]=\{(1,0,0),(1,0,1)\}$, and the revision step does not change any belief base, i.e. $E^{2} \leftrightarrow E^{3}$, so a fixed point is reached and the process stops with this profile.

Finally, for sceptical operators, we can prove that the conciliation process cannot lead to a consensus, unless a consensus already exists at start:

\section{Proposition 7}

Let $E$ be a belief profile and $\mu$ be an integrity constraint; let $\triangle$ be a merging operator and $*_{1}, \ldots, *_{n}$ be $n$ revision operators. For any integer $i$, a consensus exists for $E_{s}^{i}$ if and only if a consensus exists for $E_{s}^{0}$.

\subsection{Properties of credulous IMC operators}

Let us now turn to credulous IMC operators, and first give some general properties about such operators. 
Proposition 8

Let $K_{j}^{i}$ now denote the belief base corresponding to agent $j$ in the belief profile $E_{c}^{i}$ characterized by the initial belief profile $E$, the integrity constraint $\mu$, the merging operator $\triangle$ and $n$ revision operators $*_{1}, \ldots, *_{n}$. We have that:

- $\forall i, j K_{j}^{i+1} \models \triangle_{\mu}\left(E_{c}^{i}\right)$,

- $\forall i>0 \forall j K_{j}^{i} \models \mu$,

- $\forall i, j$, if $K_{j}^{i} \wedge \triangle_{\mu}\left(E_{c}^{i}\right)$ is consistent, then $K_{j}^{i+1} \equiv K_{j}^{i} \wedge \triangle_{\mu}\left(E_{c}^{i}\right)$.

The first item in Proposition 8 states that, during the evolution process, each base implies the previous merged base. The second item states that from the first iteration, each base implies the integrity constraints. The last one is a simple consequence of a revision property: if, at a given step, a base is consistent with the result of the merging, then the base at the next step will be the conjunction of the previous base with the merged base.

Unfortunately, the monotony property as reported in Proposition 3 does not hold in the credulous case. At that point, one can just conjecture that our credulous CHIMC operators (and the corresponding iterated merging operators) are defined for every profile:

Conjecture 1

For every credulous CHIMC operator ${ }^{*} \boldsymbol{\Delta}$ induced by a merging operator $\triangle$ based on the aggregation function Max, GMax or $\Sigma$ and for every belief profile $E$ and integrity constraint $\mu$, the sequence $\left(E_{c}^{i}\right)_{i \geq 0}$ is stationary.

This claim is supported by some empirical evidence. We have conducted exhaustive tests for profiles containing up to three bases, when the set of propositional symbols contains up to three variables. ${ }^{6}$ The following IC merging operators have been considered: $\triangle^{d_{H}, \text { Max }}, \triangle^{d_{H}, \text { GMax }}$ and $\triangle^{d_{H}, \Sigma}$. We have also conducted non-exhaustive tests when four propositional symbols are considered in the language (this leads to billions of tests). All the tested instances support the claim (stationarity is reached in less than five iterations when up to three symbols are considered, and less than ten iterations when four symbols are used).

We can nevertheless prove the stationarity of $\left(E_{c}^{i}\right)_{i \geq 0}$ for every belief profile $E$ and every integrity constraint $\mu$ when some specific IC merging operators $\triangle$ are considered, and the compatibility and homogeneity assumptions are made. In particular, for some merging operators defined from the drastic distance, it is possible to find out a non-iterative definition of the corresponding CHIMC operator, and to prove that it is defined for every profile.

PROPOSITION 9

For any profile $E=\left(K_{1}, \ldots, K_{n}\right)$ and any integrity constraint $\mu$, the credulous CHIMC operator given by ${ }^{*} \boldsymbol{\Delta}_{\mu}^{d_{D}, \operatorname{Max}}(E)=E_{c}^{*}=\left(K_{1}^{*}, \ldots, K_{n}^{*}\right)$, is s.t. for every $j$ :

$$
K_{j}^{*} \equiv \begin{cases}\mu \wedge \bigwedge_{K_{i}: K_{i} \wedge \mu \not \perp} K_{i} & \text { if consistent, else } \\ \mu \wedge K_{j} & \text { if consistent, } \\ \mu & \text { otherwise. }\end{cases}
$$

Furthermore, the resulting profile is obtained after at most two iterations (i.e. for every $i>1$, $\left.E_{c}^{i+1} \leftrightarrow E_{c}^{i}\right)$.

\footnotetext{
${ }^{6}$ Data can be obtained from the authors upon request.
} 
Proposition 10

For any profile $E=\left(K_{1}, \ldots, K_{n}\right)$ and any integrity constraint $\mu$, the credulous CHIMC operator induced by ${ }^{*} \boldsymbol{\Lambda}_{\mu}^{d_{D}, \operatorname{GMax}}(E)={ }^{*} \boldsymbol{\Lambda}_{\mu}^{d_{D}, \Sigma}(E)=E_{c}^{*}=\left(K_{1}^{*}, \ldots, K_{n}^{*}\right)$, is s.t. for every $j$ :

$$
K_{j}^{*} \equiv \begin{cases}K_{j} \wedge \triangle_{\mu}^{d_{D}, \operatorname{GMax}}(E) & \text { if consistent } \\ \triangle_{\mu}^{d_{D}, \operatorname{GMax}}(E) & \text { otherwise. }\end{cases}
$$

Furthermore, the resulting profile is obtained after at most one iteration (i.e. for every $i>0$, $\left.E_{c}^{i+1} \leftrightarrow E_{c}^{i}\right)$.

Now, whatever the credulous conciliation process terminates or not, we can prove that, like for the sceptical case, the sequence of profiles and the corresponding sequence of merged bases are equivalent w.r.t. stationarity:

\section{Proposition 11}

Let $\mathrm{E}$ be a belief profile, $\triangle$ be a merging operator and $*_{1}, \ldots, *_{n}$ be $n$ revision operators. Let $\mu$ be any integrity constraint. If the sequence $\left(E_{c}^{i}\right)_{i \geq 0}$ is stationary from $i$, then the sequence $\left(\triangle_{\mu}\left(E_{c}^{i}\right)\right)_{i \geq 0}$ is stationary from $i$. Conversely, if the sequence $\left(\triangle_{\mu}\left(E_{c}^{i}\right)\right)_{i \geq 0}$ is stationary from $i$, then the sequence $\left(E_{c}^{i}\right)_{i \geq 0}$ is stationary from $i+1$.

Let us finally consider the consensus issue. In order to illustrate the case when credulous conciliation does not lead to a consensus, let us first consider the following example as shown in Table 3.

\section{EXAMPLE 4}

Consider the profile $E=\left(K_{1}, K_{2}, K_{3}, K_{4}\right)$, with $\left[K_{1}\right]=\{(0,0,0),(0,0,1),(0,1,0)\}, \quad\left[K_{2}\right]=$ $\{(1,0,0),(1,0,1),(1,1,1)\},\left[K_{3}\right]=\{(0,0,1),(0,1,0),(0,1,1),(1,1,0)\},\left[K_{4}\right]=\{(0,1,1),(1,0,0)$, $(1,1,0),(1,1,1)\}$. There is no integrity constraint: $\mu \equiv \mathrm{T}$. Let us consider the credulous CHIMC $* \boldsymbol{\Lambda}^{d_{H}, \Sigma}$ operator induced by the merging operator $\triangle^{d_{H}, \Sigma}$. The computations are summarized in Table 3 . The resulting profile is $\left[K_{1}^{2}\right]=\{(0,0,1)\},\left[K_{2}^{2}\right]=\{(1,0,0)\}$, $\left[K_{3}^{2}\right]=\{(0,0,1)\}$ and $\left[K_{4}^{2}\right]=\{(1,0,0)\}$. The corresponding CHIM operator ${ }^{*} \triangle^{d_{H}, \Sigma}$ gives as a result a base whose set of models is $\{(0,0,0),(0,0,1),(1,0,0),(1,0,1)\}$, which is different from the result of the merging of $E$ by the underlying merging operator:

$$
\left[\triangle^{d_{H}, \Sigma}(E)\right]=\{(0,0,1),(0,1,1),(1,0,0),(1,1,0)\} .
$$

Contrastingly, there are situations where a credulous conciliation process ends with a consensus found, as illustrated by the following example (which echoes Example 1).

TABLE 3. A credulous conciliation scenario for which no consensus is reached

\begin{tabular}{l|llll|l}
\hline $\mathcal{W}$ & $K_{1}^{i}$ & $K_{2}^{i}$ & $K_{3}^{i}$ & $K_{4}^{i}$ & $E^{i}$ \\
\hline$(0,0,0)$ & $0,1,1$ & $1,1,1$ & $1,1,1$ & $1,1,1$ & $3,4,4$ \\
$(0,0,1)$ & $0,0,0$ & $1,2,2$ & $0,0,0$ & $1,1,2$ & $2,3,4$ \\
$(0,1,0)$ & $0,2,2$ & $2,2,2$ & $0,1,2$ & $1,1,2$ & $3,6,8$ \\
$(0,1,1)$ & $1,1,1$ & $1,3,3$ & $0,0,1$ & $0,0,3$ & $2,4,8$ \\
$(1,0,0)$ & $1,2,2$ & $0,0,0$ & $1,1,2$ & $0,0,0$ & $2,3,4$ \\
$(1,0,1)$ & $1,1,1$ & $0,1,1$ & $1,1,1$ & $1,1,1$ & $3,4,4$ \\
$(1,1,0)$ & $1,3,3$ & $1,1,1$ & $0,0,3$ & $0,0,1$ & $2,4,8$ \\
$(1,1,1)$ & $2,2,2$ & $0,2,2$ & $1,1,2$ & $0,1,2$ & $3,6,8$ \\
\hline
\end{tabular}


Table 4. A credulous conciliation scenario for which a consensus is reached

\begin{tabular}{l|lll|l}
\hline$\omega$ & $d\left(\omega, K_{1}^{i}\right)$ & $d\left(\omega, K_{2}^{i}\right)$ & $d\left(\omega, K_{3}^{i}\right)$ & $d_{\operatorname{Max}}\left(\omega, E^{i}\right)$ \\
\hline$(0,0,0)$ & $1,2,2$ & $1,1,2$ & $3,1,2$ & $3,2,2$ \\
$(0,0,1)$ & $0,1,1$ & $0,1,1$ & $2,1,1$ & $2,1,1$ \\
$(0,1,0)$ & $2,3,3$ & $0,1,3$ & $2,1,3$ & $2,3,3$ \\
$(0,1,1)$ & $1,2,2$ & $1,0,2$ & $1,0,2$ & $1,2,2$ \\
$(1,0,0)$ & $0,1,1$ & $2,1,1$ & $2,1,1$ & $2,1,1$ \\
$(1,0,1)$ & $0,0,0$ & $1,0,0$ & $1,0,0$ & $1,0,0$ \\
$(1,1,0)$ & $1,2,2$ & $1,0,2$ & $1,0,2$ & $1,2,2$ \\
$(1,1,1)$ & $1,1,1$ & $2,1,1$ & $0,1,1$ & $2,1,1$ \\
\hline
\end{tabular}

\section{EXAMPLE 5}

Consider the profile $E=\left(K_{1}, K_{2}, K_{3}\right)$, with $\left[K_{1}\right]=\{(0,0,1),(1,0,0),(1,0,1)\},\left[K_{2}\right]=$ $\{(0,0,1),(0,1,0)\},\left[K_{3}\right]=\{(1,1,1)\}$. There is no integrity constraint: $\mu \equiv \top$. If we consider the credulous CHIMC operator ${ }^{*} \boldsymbol{\Lambda}^{d_{H}}$, Max induced by the merging operator $\triangle^{d_{H}, \text { Max }}$, then the resulting profile is $\left[K_{1}^{*}\right]=\left[K_{2}^{*}\right]=\left[K_{3}^{*}\right]=\{(1,0,1)\}$ (see Table 4 for the computations), showing that a consensus has been reached.

\section{Iterated merging operators}

In this section, we consider CHIM operators only. Unlike other IM operators, they can be considered as true 'iterated merging operators' since they are fully specified by a given merging operator. The key issue one wants to address is to determine whether such iterated merging operators are 'good' merging ones, in the sense that they satisfy all the IC rationality postulates for merging.

Our investigation shows that the answer is negative in general: only some basic postulates are guaranteed to hold. To be more precise, our results show that when a merging operator satisfies a set of postulates, this does not imply that the corresponding iterated one satisfies the same set of postulates.

\section{Proposition 12}

Credulous and sceptical CHIM operators satisfy (IC0-IC3), (IC7) and (IC8).

Some important properties of IC merging operators are usually lost through the mergethen-revise process. So, such iterated merging operators are not as good as one could expect as merging operators: iterating the merging process does not lead to improved merging operators, as far as the rationality postulates are concerned.

Nevertheless, specific iterated merging operators (i.e. those induced by some specific merging operators $\triangle$ ) may easily satisfy additional postulates.

For operators based on the drastic distance, we have obtained the following results. As to sceptical operators, a trivialization result holds:

Corollary 1

$\triangle^{d_{D}, \mathrm{Max}}=\triangle^{* d_{D}, \mathrm{Max}}$ and $\triangle^{d_{D}, \Sigma}=\triangle^{d_{D}, \mathrm{GMax}}=\triangle^{d_{D}, \Sigma}=\triangle^{* d_{D}, \Sigma}$.

This corollary comes directly from Proposition 6. Roughly, it shows that, in the sceptical case, the iteration of the process does not change anything with respect to merging, when the underlying merging operators are $\triangle^{d_{D}, \text { Max }}$ and $\triangle^{d_{D}, \Sigma}$. Note that the logical properties of $\triangle^{d_{D}, \text { Max }}$ and $\triangle^{d_{D}, \Sigma}$ have been identified so far [23]. 
As to credulous operators, a direct corollary of Proposition 9 is:

Corollary 2

The credulous CHIM operator ${ }^{*} \triangle_{\mu}^{d_{D}, \text { Max }}$ can be defined as follows:

$$
{ }^{*} \triangle_{\mu}^{d_{D}, \operatorname{Max}}(E) \equiv \begin{cases}\mu \wedge \bigwedge_{K_{i}: K_{i} \wedge \mu \not \neq \perp} K_{i} & \text { if consistent } \\ \mu & \text { otherwise. }\end{cases}
$$

The corresponding logical properties are:

Proposition 13

The credulous CHIM operator ${ }^{*} \triangle_{\mu}^{d_{D}}$, Max satisfies (IC0-IC5), (IC7), (IC8) and (Arb). It satisfies neither (IC6) nor (Maj).

Proposition 14

The credulous CHIM operator ${ }^{*} \triangle_{\mu}^{d_{D}, \text { GMax }}={ }^{*} \triangle_{\mu}^{d_{D}, \Sigma}$ coincides with $\triangle_{\mu}^{d_{D}, \text { GMax }}=\triangle_{\mu}^{d_{D}, \Sigma}$; hence, it satisfies (IC0-IC8), (Arb) and (Maj).

Things are less easy for operators based on Dalal distance. Up to now, we did not find any equivalent, non-iterative definition for any of them. For each underlying merging operator under consideration, we gather the results about credulous/sceptical CHIM operators since it turns out that such operators satisfy the same postulates; however, the proofs are typically distinct for the two families of operators. Furthermore, since stationarity is only conjectured for credulous operators (cf. Conjecture 1), we do not have a proof that the corresponding CHIM operators are total functions. So the two following results for credulous operators are guaranteed under the conjecture of stationarity, only.

Proposition 15

The credulous (resp. sceptical) CHIM operator ${ }^{*} \triangle_{\mu}^{d_{H}, \Sigma}$ (resp. $\triangle_{\mu}^{* d_{H}, \Sigma}$ ) satisfies (IC0-IC3), (IC7), (IC8) and (Maj), but does not satisfy (IC5), (IC6) and (Arb). The satisfaction of (IC4) is an open issue.

\section{Proposition 16}

The credulous (resp. sceptical) CHIM operators ${ }^{*} \triangle_{\mu}^{d_{H}, \text { Max }}$ and ${ }^{*} \triangle_{\mu}^{d_{H}, \text { GMax }}$ (resp. ${ }^{*} \triangle_{\mu}^{d_{H}}$, Max and $\triangle_{\mu}^{* d_{H}, \mathrm{GMax}}$ ) satisfy (IC0-IC3), (IC7), (IC8), but satisfy none of (IC5), (IC6), (Maj) and (Arb). The satisfaction of (IC4) is an open issue.

Summing up the results provided in this section, there is (generally) no logical link between the initial merging operator and its iterated counterpart, in the sense that the satisfaction of a set of postulates by the former does not imply the satisfaction of the same set of postulates by the latter. The CHIM operators are shown to satisfy only poor properties with respect to merging. In particular, (IC5) and (IC6), which are important properties from an aggregation point of view since they correspond to Pareto dominance in Social Choice Theory, are usually not satisfied. This is a direct consequence of the iterative definition of such operators, and results from the impact of other agents' beliefs on the belief of each agent during the process.

Those results suggest that CHIM operators are not interesting as merging operators. Since CHIM operators compute the beliefs of the group of agents after conciliation, as the result of the negotiation modelled by the corresponding CHIMC operators, this may just reflect the fact that merging and negotiation are two distinct notions. 
In Propositions 15 and 16 we let the satisfaction of (IC4) as an open issue. This means that we do not have the proof of the result to hold (or not). Nevertheless, the satisfaction of (IC4) can be conjectured: in all the conducted experiments, we did not find any counter-example to it [as mentioned earlier, we have conducted exhaustive tests for profiles containing up to three bases, when the set of propositional symbols contains up to three variables. We have also conducted non-exhaustive tests when four propositional symbols are considered in the language (this led to billions of tests)].

\section{Related Work}

The notion of conciliation considered in this article can be viewed as a very specific form of negotiation, and as such, is related to the abundant literature in AI dealing with the latter notion. An important difference is that conciliation is concerned only with beliefs, while negotiation typically takes account for the agents' goals and the available actions. Furthermore, the issue of a negotiation process cannot be predicted in the general case: at each step the agents are free of their decisions, provided that such decisions are compatible with the chosen negotiation protocol, and the decisions made cannot be guessed (the input is not rich enough to include a representation of the model for decision making used by each agent). Contrastingly, conciliation processes can be considered as atomic decisions made by the group of agents: once the agents agree to participate to such a process, their beliefs evolve as specified by the corresponding conciliation operator.

More closely related to our work are [9-11]. In those articles, Richard Booth presents what he calls Belief Negotiation Models. Such negotiation models can be formalized as games between sources: until a coherent profile of bases is reached, at each round a contest is organized to find out the weakest belief bases, then those bases have to be logically weakened. This idea leads to numerous new interesting operators (depending of the exact meanings given to 'weakest' and 'weaken', which are the two parameters for this family). Booth is interested at the same time in the evolution of the profile (in connection to what he calls social contraction), and to the resulting merged base (the result of the Belief Negotiation Model).

In [19] a systematic study of a subclass of those operators, called Belief Game Models, is achieved. This subclass contains operators closer to merging ones than the general class which also allows for negotiation-like operators.

All those operators are close in spirit to the IMC/IM operators defined in this work. A main difference is that in the work presented in this article, the evolution of a profile does not always lead to a consensus. Scenarios where agents disagree at a final stage are allowed. So IMC operators seem more adequate to formalize interaction between agents' beliefs than Belief Game Models.

\section{Conclusion and perspectives}

In this article, we have introduced two families of conciliation processes based on an iterated merge-then-revise scheme. On this ground, conciliation operators and the associated iterated merging operators have been defined and studied.

This work calls for many perspectives. One of them concerns the stationarity conjecture related to credulous CHIMC operators.

A second perspective is about rationality postulates for conciliation operators; such postulates should reflect the fact that at the end of the conciliation process, the disagreement between the agents participating to the conciliation process is expected not to be more 
important than before; a difficulty is that it does not necessarily mean that this must be the case at each step of a conciliation process.

Another perspective is to relate this work to other approaches to conflict measurement. The point is that when a consensus is reached for a conciliation operator, one can use the number of steps needed to reach the consensus as a measure of conflict of the profiles. Such a measure could be used to compare several profiles and to determine the less conflictual ones.

Finally, it would be interesting to enrich our framework in several directions; one of them consists in studying less drastic revision behaviours, e.g. those obtained by relaxing the uniformity assumption or through the use of non-prioritized belief revision operators [14, 16, $17,26,29]$; indeed, in some situations, it can prove sensible to consider that an agent is free from rejecting a negotiation step, would it lead her to a belief state 'too far' from her original one. Another direction for further work consists in generalizing our approach to other formal settings, extending classical propositional logic; interesting candidates for such a generalization are propositional possibilistic logic [13] and the closely related $\mathrm{OCF} / \kappa$-functions [30], for which sophisticated approaches for revision and merging have been defined so far (see in particular [5-8, 31, 32]) and implemented in some cases (see in particular the SATEN system [1]).

\section{Acknowledgements}

This work has been partly supported by the Région Nord/Pas-de-Calais, the IRCICA consortium and by the European Community FEDER Program.

\section{References}

[1] Innovation Technology Research Laboratory (M. A. Williams, project leader). University of Technology, Sydney, Australia. http://magic.it.uts.edu.au/systems/saten.html

[2] C. E. Alchourrón, P. Gärdenfors, and D. Makinson. On the logic of theory change: partial meet contraction and revision functions. Journal of Symbolic Logic, 50, 510-530, 1985.

[3] C. Baral, S. Kraus, and J. Minker. Combining multiple knowledge bases. IEEE Transactions on Knowledge and Data Engineering, 3, 208-220, 1991.

[4] C. Baral, S. Kraus, J. Minker, and V. S. Subrahmanian. Combining knowledge bases consisting of first-order theories. Computational Intelligence, 8, 45-71, 1992.

[5] S. Benferhat, D. Dubois, S. Kaci, and H. Prade. Possibilistic merging and distance-based fusion of propositional information. Annals of Mathematics and Artificial Intelligence, 34, 217-252, 2002.

[6] S. Benferhat, D. Dubois, J. Lang, H. Prade, A. Saffiotti, and Ph. Smets. A general approach for inconsistency handling and merging information in prioritized knowledge bases. In Proceedings of the Sixth International Conference on the Principles of Knowledge Representation and Reasoning (KR'98), pp. 466-477, 1998.

[7] S. Benferhat and S. Kaci. Logical representation and fusion of prioritized information based on guaranteed possibility measures: application to the distance-based merging of classical bases. Artificial Intelligence, 148, 291-333, 2003.

[8] S. Benferhat, S. Kaci, D. Le Berre, and M.-A. Williams. Weakening conflicting information for iterated revision and knowledge integration. Artificial Intelligence, 153, 339-371, 2004.

[9] R. Booth. A negociation-style framework for non-prioritised revision. In Proceedings of the Eighth Conference on Theoretical Aspects of Rationality and Knowledge (TARK'01), pp. 137-150, 2001. 
[10] R. Booth. Social contraction and belief negociation. In Proceedings of the Eighth Conference on Principles of Knowledge Representation and Reasoning (KR'02), pp. 374-384, 2002.

[11] R. Booth. Social contraction and belief negotiation. Information Fusion, 7, 19-34, 2006.

[12] M. Dalal. Investigations into a theory of knowledge base revision: preliminary report. In Proceedings of the Seventh American National Conference on Artificial Intelligence (AAAI'88), pp. 475-479, 1988.

[13] D. Dubois, J. Lang, and H. Prade. Possibilistic logic. In Handbook of Logic in Artificial Intelligence and Logic Programming, Volume 3: Nonmonotonic Reasoning and Uncertain Reasoning, D. Gabbay, C. J. Hogger and J. A. Robinson, eds, pp. 439-513. Oxford University Press, Oxford, 1994.

[14] E. Fermé and S. O. Hansson. Selective revision. Studia Logica, 63, 331-342, 1999.

[15] P. Gärdenfors. Knowledge in Flux. MIT Press, Cambridge, 1988.

[16] S. O. Hansson. Semi-revision. Journal of Applied Non-Classical Logic, 7, 151-175, 1997.

[17] S. O. Hansson. What's new isn't always best. Theoria, 63, 1-13, 1998. (Special issue on non-prioritized belief revision).

[18] H. Katsuno and A. O. Mendelzon. Propositional knowledge base revision and minimal change. Artificial Intelligence, 52, 263-294, 1991.

[19] S. Konieczny. Belief base merging as a game. Journal of Applied Non-Classical Logics, 14, 275-294, 2004.

[20] S. Konieczny, J. Lang, and P. Marquis. DA ${ }^{2}$ merging operators. Artificial Intelligence, 157, 49-79, 2004.

[21] S. Konieczny and R. P. Pérez. On the logic of merging. In Proceedings of the Sixth International Conference on Principles of Knowledge Representation and Reasoning (KR'98), pp. 488-498, 1998.

[22] S. Konieczny and R. P. Pérez. Merging with integrity constraints. In Proceedings of the Fifth European Conference on Symbolic and Quantitative Approaches to Reasoning with Uncertainty (ECSQARU'99), LNAI 1638, pp. 233-244, 1999.

[23] S. Konieczny and R. P. Pérez. Merging information under constraints: a logical framework. Journal of Logic and Computation, 12, 773-808, 2002.

[24] P. Liberatore and M. Schaerf. Arbitration (or how to merge knowledge bases). IEEE Transactions on Knowledge and Data Engineering, 10, 76-90, 1998.

[25] J. Lin and A. O. Mendelzon. Knowledge base merging by majority. In Dynamic Worlds: From the Frame Problem to Knowledge Management. Kluwer Academic Publishers, Dordrecht, 1999.

[26] D. Makinson. Screened revision. Theoria, 63, 14-23, 1998 (special issue on nonprioritized belief revision).

[27] P. Z. Revesz. On the semantics of theory change: arbitration between old and new information. In Proceedings of the Twelfth ACM SIGACT-SIGMOD-SIGART Symposium on Principles of Databases (PODS'93), pp. 71-92, 1993.

[28] P. Z. Revesz. On the semantics of arbitration. International Journal of Algebra and Computation, 7, 133-160, 1997.

[29] K. Schlechta. Non-prioritized belief revision based on distances between models. Theoria, 63, 34-53, 1998 (special issue on non-prioritized belief revision).

[30] W. Spohn. Ordinal conditional functions: a dynamic theory of epistemic states. In Causation in Decision, Belief Change, and Statistics, vol. 2, W. L. Harper and B. Skyrms, eds, 1988. 
[31] M.-A. Williams. Transmutations of knowledge systems. In Proceedings of the Fourth International Conference on the Principles of Knowledge Representation and Reasoning (KR'94), pp. 619-629, 1994.

[32] M.-A. Williams. Iterated theory base change: a computational model. In Proceedings of the Fourteenth International Joint Conference on Artificial Intelligence (IJCAI'95), pp. 1541-1549, 1995.

Received 8 February 2006

\section{Appendix}

\section{Proofs}

Proof of Proposition 2. Let us first consider the credulous case. If there is a consensus for $E_{c}^{i}$, then $\mu \wedge \wedge E_{c}^{i}$ is consistent. From the (IC2) property of the merging operator, we have that $\triangle_{\mu}\left(E_{c}^{i}\right) \equiv \mu \wedge \wedge E_{c}^{i}$. Hence for any $K_{j}^{i}$, we have $K_{j}^{i} \wedge \triangle_{\mu}\left(E_{c}^{i}\right)$ consistent. Since $*_{j}$ is an AGM revision operator, by $(\mathbf{R 2})$ we have that $K_{j}^{i} *_{j} \triangle_{\mu}\left(E_{c}^{i}\right) \equiv K_{j}^{i} \wedge \triangle_{\mu}\left(E_{c}^{i}\right)$. Since $\triangle_{\mu}\left(E_{c}^{i}\right) \models K_{j}^{i}$, we have $K_{j}^{i} *_{j} \triangle_{\mu}\left(E_{c}^{i}\right) \equiv \triangle_{\mu}\left(E_{c}^{i}\right)$. So the new profile is $E_{c}^{i+1}=\left(\triangle_{\mu}\left(E_{c}^{i}\right), \ldots, \triangle_{\mu}\left(E_{c}^{i}\right)\right)$. From (IC2), the result of the merging of this profile is $\triangle_{\mu}\left(E_{c}^{i+1}\right) \equiv \triangle_{\mu}\left(E_{c}^{i}\right)$. Furthermore, since for any $j$, we have $K_{j}^{i+1} \equiv \triangle_{\mu}\left(E_{c}^{i}\right)$ we have $K_{j}^{i+1} \wedge \triangle_{\mu}\left(E_{c}^{i}\right) \equiv K_{j}^{i+1}$ which is consistent. From (R2) we get that $K_{j}^{i+2} \equiv K_{j}^{i+1}$, hence $E_{c}^{i+2} \leftrightarrow E_{c}^{i+1}$. So $E_{c}^{*}=E_{c}^{i+1}$. The proof is similar in the sceptical case, mutatis mutandis (the key observation is that we also have $\triangle_{\mu}\left(E_{s}^{i}\right) *_{j} K_{j}^{i} \equiv$ $\left.K_{j}^{i} \wedge \triangle_{\mu}\left(E_{s}^{i}\right) \equiv \triangle_{\mu}\left(E_{s}^{i}\right)\right)$.

Proof of Proposition 3. By definition $K_{j}^{i+1}=\triangle_{\mu}\left(E_{s}^{i}\right) *_{j} K_{j}^{i}$. Since $*_{j}$ is an AGM revision operator, from postulate (R1), we have that $K_{j}^{i+1} \models K_{j}^{i}$.

Proof of Proposition 4. The first implication is straightforward by Definition 12. As to the second one, suppose that $\triangle_{\mu}\left(E_{s}^{i+1}\right) \equiv \triangle_{\mu}\left(E_{s}^{i}\right)$. By definition of the AGM revision operator, and thanks to Katsuno and Mendelzon representation theorem [18] we have that $\left[K_{j}^{i+1}\right]=\min \left(\left[K_{j}^{i}\right], \leq_{\triangle_{\mu}\left(E_{s}^{i}\right)}^{j}\right)$, where $\leq_{\triangle_{\mu}\left(E_{s}^{i}\right)}^{j}$ is the total pre-order associated to $\triangle_{\mu}\left(E_{s}^{i}\right)$ by the faithful assignment of the revision operator $*_{j}$. Similarly $\left[K_{j}^{i+2}\right]=\min \left(\left[K_{j}^{i+1}\right], \leq_{\triangle_{\mu}\left(E_{s}^{i+1}\right)}\right)$. But, since by assumption, $\triangle_{\mu}\left(E_{s}^{i+1}\right) \equiv \triangle_{\mu}\left(E_{s}^{i}\right)$, this gives $\left[K_{j}^{i+2}\right]=\min \left(\min \left(\left[K_{j}^{i}\right]\right.\right.$, $\left.\left.\leq_{\triangle_{\mu}\left(E_{s}^{i}\right)}^{j}\right), \leq_{{ }_{\mu}\left(E_{s}^{i}\right)}^{j}\right)=\min \left(\left[K_{j}^{i}\right], \leq_{{ }_{{ }_{\mu}}\left(E_{s}^{i}\right)}^{j}\right)=\left[K_{j}^{i+1}\right]$. So $K_{j}^{i+2} \equiv K_{j}^{i+1}$. Hence $E_{s}^{i+1} \leftrightarrow E_{s}^{i+2}$.

Proof of Proposition 5. Let us consider the sequence $\left(u_{i}\right)_{i \geq 0}$, where $u_{i}=\sum_{j} \#\left(\left[K_{j}^{i}\right]\right)$. From Proposition 3 we know that this sequence of positive integers is non-increasing. So there exists a least integer $i$ s.t. $u_{i}=u_{i+1}$.

From Proposition 3, we know that for every $i, j$ every model of $K_{j}^{i+1}$ is a model of $K_{j}^{i}$, and from Proposition 4 we have that if $E_{s}^{i+1} \leftrightarrow E_{s}^{i}$ for some $i$ then the fixed point is reached. For every $j<i$, we have $u_{j}>u_{j+1}$. We also know that when the fixed point is reached, there is at least one model for each base; this gives the upper bound $\sum_{K \in E} \#([K])-\#(E)$.

Proof of Proposition 6. For any of the operators under consideration, if $\mu \wedge \wedge E$ is consistent, then from postulate (IC2) we know that the result of the merging is this conjunction, and from (R1), (R2) and (R3) of the corresponding revision operator we have that for each $j K_{j}^{1} \equiv \mu \wedge \wedge E$. So the fixed point is reached after one iteration, and $K_{j}^{*} \equiv \mu \wedge \wedge E$. 
If $\mu \wedge \bigwedge E$ is not consistent, then:

- For $\triangle^{d_{D}, \text { Max }}$, we have $\triangle_{\mu}^{d_{D}, \text { Max }}(E) \equiv \mu$. So by the properties of the corresponding revision operator, for each $j, K_{j}^{1} \equiv K_{j} \wedge \mu$ if this is consistent, and $K_{j}^{1} \equiv K_{j}$ otherwise. So $\triangle_{\mu}^{d_{D}, \operatorname{Max}}\left(E^{1}\right) \equiv \triangle_{\mu}^{d_{D}}$, Max $(E)$, and the fixed point is reached.

- For $\triangle^{d_{D}, \Sigma}=\triangle^{d_{D}, \text { GMax }}$, we have for each $j$, if $\triangle_{\mu}^{d_{D}, \Sigma}(E) \wedge K_{j}$ is consistent, then $K_{j}^{1} \equiv \triangle_{\mu}^{d_{D}, \Sigma}(E) \wedge K_{j}$, else $K_{j}^{1} \equiv K_{j}$. It is easy to show that $\triangle_{\mu}^{d_{D}, \Sigma}\left(E^{1}\right) \equiv \triangle_{\mu}^{d_{D}, \Sigma}(E)$, and that the fixed point is reached at this stage.

Proof of Proposition 7. This result is a straightforward consequence of Proposition 3. From Proposition 3 we have $K_{j}^{i+1} \models K_{j}^{i}$. Hence, if there exists a rank $i$ such that $K_{1}^{i} \wedge \ldots \wedge K_{n}^{i}$ is consistent, then $K_{1}^{0} \wedge \ldots \wedge K_{n}^{0}$ is consistent as well. Therefore, there is a consensus for $E$ at the start.

Proof of Proposition 8. These results are direct consequences of the fact that $\triangle$ is an IC merging operator and $*_{1}, \ldots, *_{n}$ are AGM revision operators:

- By definition of credulous operators, $K_{j}^{i+1} \equiv K_{j}^{i} *_{j} \triangle_{\mu}\left(E_{c}^{i}\right)$. Since $*_{j}$ is an AGM revision operator, it satisfies (R1), so $K_{j}^{i+1} \models \triangle_{\mu}\left(E_{c}^{i}\right)$.

- From (IC0) we have $\triangle_{\mu}\left(E_{c}^{i}\right) \models \mu$; we also have $\forall i, j K_{j}^{i+1} \models \triangle_{\mu}\left(E_{c}^{i}\right)$; hence $\forall i, j K_{j}^{i+1} \models \mu$.

- By definition of credulous operators, $K_{j}^{i+1} \equiv K_{j}^{i} *_{j} \triangle_{\mu}\left(E_{c}^{i}\right)$. From (R2) we have that if $K_{j}^{i} \wedge \triangle_{\mu}\left(E_{c}^{i}\right)$ is consistent, then $K_{j}^{i+1} \equiv K_{j}^{i} \wedge \triangle_{\mu}\left(E_{c}^{i}\right)$.

Proof of Proposition 9.

- If $\mu \wedge \wedge E$ is consistent then, by (IC2) we have that $\triangle_{\mu}^{d_{D}, \operatorname{Max}}(E) \equiv \mu \wedge \wedge E$. Then for every $j, K_{j}^{1} \equiv K_{j} * \triangle \triangle_{\mu}^{d_{D}, \operatorname{Max}}(E) \equiv \triangle_{\mu}^{d_{D}, \operatorname{Max}}(E)$ (by (R1)). So the fixed point is reached after one iteration, and for every $j, K_{j}^{*} \equiv \mu \wedge \wedge E$.

- If $\mu \wedge \wedge E$ is not consistent, then by definition of the operator, $\triangle_{\mu}^{d_{D}, \operatorname{Max}}(E) \equiv \mu$. Then for every $K_{j}$ there are two cases for the revision. Either $K_{j} \wedge \mu$ is consistent, then by (R1), this is the result of the revision, and $K_{j}^{1} \equiv K_{j} \wedge \mu$. Or $K_{j} \wedge \mu$ is not consistent and by definition of the revision $K_{j}^{1} \equiv \mu$. Now let us compute $\triangle_{\mu}^{d_{D}, \operatorname{Max}}\left(E^{1}\right)$ :

- If $\mu \wedge \wedge E^{1}$ is consistent then, by (IC2) we have that $\triangle_{\mu}^{d_{D}, \operatorname{Max}}\left(E^{1}\right) \equiv \mu \wedge \wedge E^{1} \equiv$ $\mu \wedge \bigwedge_{K_{i}: K_{i} \wedge \mu \neq \perp} K_{i}$. So the fixed point is reached after two iterations, since for every $j$, $K_{j}^{2} \stackrel{K_{i}: K_{i} \wedge \mu \not \neq \perp}{\equiv} K_{j}^{*} \equiv \mu \wedge \bigwedge_{K_{i}: K_{i} \wedge \mu \not \neq \perp} K_{i}$.

- If $\mu \wedge \wedge E^{1}$ is not consistent then by definition of the operator, $\triangle_{\mu}^{d_{D}, K_{i} \wedge \mu \neq \operatorname{Max}}\left(E^{2}\right) \equiv \mu$. Since every $K_{j}^{1}$ is consistent with $\mu$, by (R1), this is the result of the revision, and $K_{j}^{2} \equiv K_{j}^{1} \wedge \mu$. Since $K_{j}^{1} \models \mu$, we have that $K_{j}^{2} \equiv K_{j}^{1} \equiv K_{j}^{*}$. So we have that for all $j$ either $K_{j}^{*} \equiv K_{j} \wedge \mu$ if consistent, otherwise $K_{j}^{*} \equiv \mu$, and the fixed point is reached after one iteration.

Proof of Proposition 10. If $\mu \wedge \wedge E$ is consistent, then by (IC2) we have that $\triangle_{\mu}^{d_{D}, \operatorname{GMax}}(E) \equiv \mu \wedge \wedge E$. Then for every $j, K_{j}^{1} \equiv K_{j} *_{\triangle} \triangle_{\mu}^{d_{D}, \operatorname{GMax}}(E) \equiv \triangle_{\mu}^{d_{D}, \operatorname{GMax}}(E)$ (consequence of (R1)). So the fixed point is reached after one iteration, and for every $j$, $K_{j}^{*} \equiv \mu \wedge \wedge_{j} K_{j}$

Suppose now that $\mu \wedge \wedge E$ is not consistent. $\left[\Delta_{\mu}\left(E^{0}\right)\right]=\left\{\omega \models \mu: \nexists \omega^{\prime}: \#\right.$ $\left.\left(\left\{j: \omega^{\prime} \models K_{j}^{0}\right\}\right)>\#\left(\left\{j: \omega \models K_{j}^{0}\right\}\right)\right\}$. Using the notation $a=\max _{\omega \models \mu} \#\left(\left\{j: \omega \models K_{j}^{0}\right\}\right.$, we 
get that $\left[\Delta_{\mu}\left(E^{0}\right)\right]=\left\{\omega \models \mu: \#\left(\left\{j: \omega \models K_{j}^{0}\right\}\right)=a\right\}$. For each $j$, we have $K_{j}^{1} \equiv K_{j}^{0} * \Delta$ $\triangle_{\mu}^{d_{D}, \operatorname{GMax}}\left(E^{0}\right)$, i.e.:

- if $K_{j}^{0} \wedge \triangle_{\mu}^{d_{D}, \operatorname{GMax}}\left(E^{0}\right) \not \equiv \perp$ then $K_{j}^{1} \equiv K_{j}^{0} \wedge \triangle_{\mu}^{d_{D}, \operatorname{GMax}}\left(E^{0}\right)$.

- if $K_{j}^{0} \wedge \triangle_{\mu}^{d_{D}, \operatorname{GMax}}\left(E^{0}\right) \equiv \perp$ then by definition of the corresponding revision operator, we get $K_{j}^{1} \equiv \triangle_{\mu}^{d_{D}}$,GMax $\left(E^{0}\right)$. Let $b$ denote the number of bases in this case.

It is easy to see that $\left[\Delta_{\mu}\left(E^{1}\right)\right]=\left\{\omega \models \mu: \nexists \omega^{\prime}: \#\left(\left\{j: \omega^{\prime} \models K_{j}^{1}\right\}\right)>\#\left(\left\{j: \omega \models K_{j}^{1}\right\}\right)\right\}=\{\omega \models \mu: \#$ $\left.\left(\left\{j: \omega \models K_{j}^{1}\right\}\right)=a+b\right\}$. So $\left[\Delta_{\mu}\left(E^{1}\right)\right]=\left[\Delta_{\mu}\left(E^{0}\right)\right]$. Then the revision of each base lets it unchanged, so for every $j K_{j}^{2} \equiv K_{j}^{1}$, which means that $E^{2} \leftrightarrow E^{1}$, i.e. $E^{1}=E^{*}$.

Proof of Proposition 11. The first implication is straightforward by Definition 13. As to the second implication, suppose that $\triangle_{\mu}\left(E_{c}^{i+1}\right) \equiv \triangle_{\mu}\left(E_{c}^{i}\right)$. For any $j$, we have $K_{j}^{i+2} \equiv$ $K_{j}^{i+1} *_{j} \triangle_{\mu}\left(E_{c}^{i+1}\right) \equiv K_{j}^{i+1} *_{j} \triangle_{\mu}\left(E_{c}^{i}\right)$.

From Proposition 8, we have that $K_{j}^{i+1} \models \triangle_{\mu}\left(E_{c}^{i}\right)$. Hence, using property (R2) of the revision operator, we get $K_{j}^{i+2} \equiv K_{j}^{i+1}$. Therefore, $E_{c}^{i+1} \leftrightarrow E_{c}^{i+2}$.

Proof of Proposition 12. Let us first consider credulous operators:

(IC0) Since the underlying merging operator is an IC merging operator, it satisfies (IC0), so $\forall i, \triangle_{\mu}\left(E^{i}\right) \models \mu$, and in particular for $E^{i}=E^{*}$.

(IC1) If $\mu$ is consistent, then since $\triangle$ is an IC merging operator, $\triangle_{\mu}\left(E^{*}\right)$ is consistent.

(IC2) Suppose that $\mu \wedge \bigwedge E^{0} \not \equiv \perp$ and that $\triangle$ is an IC merging operator. From (IC2) we have $\Delta_{\mu}\left(E^{0}\right) \equiv \mu \wedge \wedge E^{0}$. So for a given $j, K_{j}^{1} \equiv \Delta_{\Delta_{\mu}\left(E^{0}\right)}\left(K_{j}^{0}\right) \equiv \mu \wedge \wedge E^{0}$. Similarly, since $\mu \wedge \wedge E^{1} \equiv \mu \wedge \wedge E^{0} \not \equiv \perp$, we have $\Delta_{\mu}\left(E^{1}\right) \equiv \mu \wedge \wedge E^{1} \equiv \mu \wedge \wedge E^{0} \equiv \Delta_{\mu}\left(E^{0}\right)$. Then $K_{j}^{2} \equiv \Delta_{\Delta_{\mu}\left(E^{1}\right)}\left(K_{j}^{1}\right) \equiv \mu \wedge \wedge E^{1} \equiv \mu \wedge \wedge E^{0} \equiv K_{j}^{1}$.

(IC3) Straightforward from the definition of credulous operators and the IC merging properties of the merging operator.

(IC7) If $\Delta$ is an IC merging operator, then for every profile $E, \Delta_{\mu_{1}}(E) \wedge \mu_{2} \models \Delta_{\mu_{1} \wedge \mu_{2}}(E)$. So, for a given profile $E$, we get $\Delta_{\mu_{1}}\left(E^{*}\right) \wedge \mu_{2} \models \Delta_{\mu_{1} \wedge \mu_{2}}\left(E^{*}\right)$.

(IC8) If $\Delta$ is an IC merging operator, then for every profile $E$, if $\Delta_{\mu_{1}}(E) \wedge \mu_{2}$ is consistent, then $\Delta_{\mu_{1} \wedge \mu_{2}}(E) \models \Delta_{\mu_{1}}(E) \wedge \mu_{2}$. With $E=E^{*}$, this shows that (IC8) holds for credulous operators.

Let us now consider sceptical operators:

(IC0) Since the underlying merging operator is an IC merging operator, it satisfies (IC0), so $\forall i, \triangle_{\mu}\left(E^{i}\right) \models \mu$, and in particular for $E^{i}=E^{*}$.

(IC1) If $\mu$ is consistent, then since $\triangle$ is an IC merging operator, $\triangle_{\mu}\left(E^{*}\right)$ is consistent.

(IC2) Suppose that $\mu \wedge \bigwedge E^{0} \not \equiv \perp$ and that $\triangle$ is an IC merging operator. We know that $\wedge E^{0}$ is consistent. Since $\triangle$ satisfies (IC2) we have $\Delta_{\mu}\left(E^{0}\right) \equiv \mu \wedge \wedge E^{0}$. So, for a given $j$, we have $K_{j}^{1} \equiv \Delta_{K_{j}^{0}}\left(\Delta_{\mu}\left(E^{0}\right)\right) \equiv \mu \wedge \wedge E^{0}$. Similarly, since $\mu \wedge \wedge E^{1} \equiv$ $\mu \wedge \wedge E^{0} \not \equiv \perp$, we have $\Delta_{\mu}\left(E^{1}\right) \equiv \mu \wedge \wedge E^{1} \equiv \mu \wedge \wedge E^{0} \equiv \Delta_{\mu}\left(E^{0}\right)$. Then $K_{j}^{2} \equiv \Delta_{K_{j}^{1}}$ $\left(\Delta_{\mu}\left(E^{1}\right)\right) \equiv \mu \wedge \wedge E^{1} \equiv \mu \wedge \wedge E^{0} \equiv K_{j}^{1}$

(IC3) Straightforward from the definition of credulous operators and the IC merging properties of the merging operator.

(IC7) If $\Delta$ is an IC merging operator, then for every profile $E, \Delta_{\mu_{1}}(E) \wedge \mu_{2} \vDash \Delta_{\mu_{1} \wedge \mu_{2}}(E)$. Just take $E=E^{*}$. 
(IC8) If $\Delta$ is an IC merging operator, then for every profile $E$, if $\Delta_{\mu_{1}}(E) \wedge \mu_{2}$ is consistent, then $\Delta_{\mu_{1} \wedge \mu_{2}}(E) \models \Delta_{\mu_{1}}(E) \wedge \mu_{2}$. Just take $E=E^{*}$.

Proof of Proposition 13. The definition is a straightforward consequence of Proposition 9. As to the postulates, for the ease of reading, we use indexes $j_{1}$ for the bases $K_{j_{1}}$ of $E_{1}$, and indexes $j_{2}$ for the bases $K_{j_{2}}$ of $E_{2}$.

(IC0-IC3), (IC7), (IC8) see Proposition 12.

(IC4) Assume that $K_{1} \models \mu, K_{2} \models \mu$. Suppose that $\mu \not \equiv \perp$ (if it is not the case the result holds trivially). We can compute ${ }^{*} \Delta_{\mu}^{d_{D}, \operatorname{Max}}\left(\left(K_{1}, K_{2}\right)\right)$ from Proposition 9:

- If $\mu \wedge \bigwedge_{j: K_{j} \wedge \mu \neq \perp} K_{j} \not \equiv \perp$, then $\mu \wedge \wedge E$ is consistent. Now by (IC2) (cf. Proposition 12), we get : ${ }^{*} \Delta_{\mu}^{d_{D}, \operatorname{Max}}\left(\left(K_{1}, K_{2}\right)\right) \wedge K_{1} \equiv K_{1} \wedge K_{2} \equiv{ }^{*} \Delta_{\mu}^{d_{D}, \operatorname{Max}}\left(\left(K_{1}, K_{2}\right)\right) \wedge K_{2} \not \forall \perp$.

- Otherwise, ${ }^{*} \Delta_{\mu}^{d_{D}, \operatorname{Max}}\left(\left(K_{1}, K_{2}\right)\right) \equiv \mu$. Hence ${ }^{*} \Delta_{\mu}^{d_{D}, \operatorname{Max}}\left(\left(K_{1}, K_{2}\right)\right) \wedge K_{1} \equiv K_{1} \not \forall \perp$ and ${ }^{*} \Delta_{\mu}^{d_{D}, \operatorname{Max}}\left(\left(K_{1}, K_{2}\right)\right) \wedge K_{2} \equiv K_{2} \not \forall \perp$.

(IC5) From Proposition 9 we can compute ${ }^{*} \Delta_{\mu}^{d_{D}, \operatorname{Max}}\left(E_{1}\right),{ }^{*} \Delta_{\mu}^{d_{D}, \operatorname{Max}}\left(E_{2}\right)$, and ${ }^{*} \Delta_{\mu}^{d_{D}, \operatorname{Max}}\left(E_{1} \sqcup E_{2}\right)$ :

- If $\mu \wedge \bigwedge_{j_{1}: \mu \wedge K_{j_{1}} \neq \perp} K_{j_{1}}$ is consistent, and if $\mu \wedge \bigwedge_{j_{2}: \mu \wedge K_{j_{2}} \neq \perp} K_{j_{2}}$ is also consistent, then we get ${ }^{*} \Delta_{\mu}^{d_{D}, \operatorname{Max}}\left(E_{1}\right) \equiv \mu \wedge \bigwedge_{j_{1}: \mu \wedge K_{j_{1}} \neq \perp} K_{j_{1}}$, and ${ }^{*} \Delta_{\mu}^{d_{D}, \operatorname{Max}}\left(E_{2}\right) \equiv \mu \wedge \bigwedge_{j_{2}: \mu \wedge K_{j_{2}} \neq \perp} K_{j_{2}}$. If $\mu \wedge \bigwedge_{j: \mu \wedge K_{j} \neq \perp} K_{j}$ is consistent, then ${ }^{*} \Delta_{\mu}^{d_{D}, \operatorname{Max}}\left(E_{1} \sqcup E_{2}\right) \equiv \mu \wedge \bigwedge_{j: \mu \wedge K_{j} \neq \perp} K_{j} \equiv$ ${ }^{*} \Delta_{\mu}^{d_{D}, \operatorname{Max}}\left(E_{1}\right) \wedge{ }^{*} \Delta_{\mu}^{d_{D}, \operatorname{Max}}\left(E_{2}\right)$. Otherwise, ${ }^{*} \Delta_{\mu}^{d_{D}, \operatorname{Max}}\left(E_{1}\right) \wedge{ }^{*} \Delta_{\mu}^{d_{D}, \operatorname{Max}}\left(E_{2}\right)$ is not consistent, so (IC5) holds.

- Otherwise, $\mu \wedge \bigwedge_{j: \mu \wedge K_{i} \neq \perp \perp} K_{j}$ is not consistent. Hence ${ }^{*} \Delta_{\mu}^{d_{D}, \operatorname{Max}}\left(E_{1} \sqcup E_{2}\right) \equiv \mu$. Since ${ }^{*} \Delta_{\mu}^{d_{D}, \operatorname{Max}}\left(E_{1}\right) \wedge{ }^{*} \Delta_{\mu}^{d_{D}, \operatorname{Max}}\left(E_{2}\right) \models \mu$ (from postulate (IC0), cf. Proposition 12), (IC5) also holds.

(IC6) Suppose that $E_{1}$ and $E_{2}$ are such that $\mu \wedge \bigwedge_{j_{1}: \mu \wedge K_{j_{1}} \neq \perp} K_{j_{1}}$ is not consistent, and $\mu \wedge \bigwedge_{j_{2}: \mu \wedge K_{j_{2}} \neq \perp} K_{j_{2}}$ is consistent, but not equivalent to $\mu$. In this case, we have ${ }^{*} \Delta_{\mu}^{d_{D}, \operatorname{Max}}\left(E_{1}\right) \equiv \mu$ and $*^{*} \Delta_{\mu}^{d_{D}, \operatorname{Max}}\left(E_{2}\right) \equiv \mu \wedge \bigwedge_{j_{2}: \mu \wedge K_{j_{2}} \neq \perp} K_{j_{2}}$, so ${ }^{*} \Delta_{\mu}^{d_{D}, \operatorname{Max}}\left(E_{1}\right) \wedge$ ${ }^{*} \Delta_{\mu}^{d_{D}, \operatorname{Max}}\left(E_{2}\right) \equiv{ }^{*} \Delta_{\mu}^{d_{D}, \operatorname{Max}}\left(E_{2}\right)$ (from (IC0)). Since $\mu \wedge \bigwedge_{j_{2}: \mu \wedge K_{j_{2}} \neq \perp} K_{j_{2}}$ is consistent, but not equivalent to $\mu$, we have $\mu \not *^{*} \Delta_{\mu}^{d_{D}}\left(E_{1}\right) \wedge{ }^{*} \Delta_{\mu}^{d_{D}, \operatorname{Max}}\left(E_{2}\right)$. Moreover, $\mu \wedge \bigwedge_{j: \mu \wedge K_{j} \neq \perp \perp} K_{j}$ is not consistent (because of $E_{1}$ ), so ${ }^{*} \Delta_{\mu}^{d_{D}, \operatorname{Max}}\left(E_{1} \sqcup E_{2}\right) \equiv \mu$. So (IC6) does not hold.

(Maj) Let us consider the following counter-example. We consider a language with two propositional symbols $a, b$ and two bases $K_{1} \equiv \neg a \wedge \neg b$ and $K_{2} \equiv \neg a \wedge b$, with $\mu \equiv \top$, we have ${ }^{*} \Delta_{\mu}^{d_{D}, \operatorname{Max}}(E_{1} \sqcup \underbrace{E_{2} \sqcup \ldots \sqcup E_{2}}_{m}) \equiv{ }^{*} \Delta_{\mu}^{d_{D}, \operatorname{Max}}\left(E_{1} \sqcup E_{2}\right) \equiv \top$, and
${ }^{*} \Delta_{\mu}^{d_{D}, \operatorname{Max}}\left(E_{2}\right) \equiv \neg a \wedge b$.

(Arb) Suppose that ${ }^{*} \Delta_{\mu_{1}}^{d_{D}, \operatorname{Max}}\left(K_{1}\right) \equiv{ }^{*} \Delta_{\mu_{2}}^{d_{D}, \operatorname{Max}}\left(K_{2}\right),{ }^{*} \Delta_{\mu_{1} \Leftrightarrow \neg \mu_{2}}^{d_{D}, \operatorname{Max}}\left(K_{1} \sqcup K_{2}\right) \equiv\left(\mu_{1} \Leftrightarrow \neg \mu_{2}\right), \mu_{1} \not \forall \mu_{2}$, and that $\mu_{2} \not \forall \mu_{1}$. Then we have $\mu_{1} \wedge K_{1} \not \equiv \perp$ : otherwise this would mean ${ }^{*} \Delta_{\mu_{1}}^{d_{D}, \operatorname{Max}}\left(K_{1}\right) \equiv \mu_{1}$, and since ${ }^{*} \Delta_{\mu_{1}}^{d_{D}, \operatorname{Max}}\left(K_{1}\right) \equiv{ }^{*} \Delta_{\mu_{2}}^{d_{D}, \operatorname{Max}}\left(K_{2}\right)$, we would get $\mu_{1} \models \mu_{2}$. Similarly, we have $\mu_{2} \wedge K_{2} \not \equiv \perp$. With Proposition 9 we obtain $\mu_{1} \wedge K_{1} \equiv$ ${ }^{*} \Delta_{\mu_{1}}^{d_{D}, \operatorname{Max}}\left(K_{1}\right) \equiv{ }^{*} \Delta_{\mu_{2}}^{d_{D}, \operatorname{Max}}\left(K_{2}\right) \equiv \mu_{2} \wedge K_{2}$. This leads $\quad$ to $\quad \mu_{1} \wedge \mu_{2} \wedge \quad K_{1} \wedge K_{2} \not \equiv \perp$. To show that ${ }^{*} \Delta_{\mu_{1} \vee \mu_{2}}^{\mu_{D}, \operatorname{Max}}\left(K_{1} \sqcup K_{2}\right) \equiv{ }^{*} \Delta_{\mu_{1}}^{d_{D}, \operatorname{Max}}\left(K_{1}\right)$, it remains to show that $\left(\mu_{1} \oplus \mu_{2}\right) \wedge$ $K_{1} \wedge K_{2} \equiv \perp$. It is enough to notice that $\mu_{1} \wedge \neg \mu_{2} \wedge K_{1} \wedge K_{2} \equiv \mu_{2} \wedge \neg \mu_{2} \wedge K_{2} \equiv \perp$, and similarly for $\neg \mu_{1} \wedge \mu_{2} \wedge K_{1} \wedge K_{2}$. 


\section{Conciliation through Iterated Belief Merging}

Proof of Proposition 14. The first point easily comes from Proposition 10. The IC properties of ${ }^{*} \triangle_{\mu}^{d_{D}, \text { GMax }}=\triangle_{\mu}^{d_{D}, \Sigma}$ are shown in [23].

Proof of Proposition 15. For the credulous CHIM operator ${ }^{*} \triangle_{\mu}^{d_{H}, \Sigma}$ the proofs are:

(IC0-IC3), (IC7), (IC8) see Proposition 12.

(IC5) The example in the following table is a counter-example to (IC5). Consider a language with three propositional symbols $\{a, b, c\}$ and the profiles $E_{1}=\left(K_{1}, K_{2}\right)$, $E_{2}=\left(K_{3}, K_{4}\right)$ and $E=E_{1} \sqcup E_{2}$.

\begin{tabular}{l|llll|l||ll|l||ll|l}
\hline $\mathcal{W}$ & $K_{1}^{i}$ & $K_{2}^{i}$ & $K_{3}^{i}$ & $K_{4}^{i}$ & $E^{i}$ & $K_{1}^{i}$ & $K_{2}^{i}$ & $E_{1}^{i}$ & $K_{3}^{i}$ & $K_{4}^{i}$ & $E_{2}^{i}$ \\
\hline$(0,0,0)$ & $2,1,1$ & $3,2,2$ & $0,1,1$ & $1,1,1$ & $6,5,5$ & 2,1 & 3,2 & 5,3 & $0,0,0$ & $1,1,1$ & $1,1,1$ \\
$(0,0,1)$ & $1,0,0$ & $2,1,1$ & $1,0,0$ & $0,0,0$ & $4,1,1$ & 1,0 & 2,1 & 3,1 & $1,1,1$ & $0,0,0$ & $1,1,1$ \\
$(0,1,0)$ & $1,0,2$ & $2,3,3$ & $1,0,2$ & $0,0,2$ & $4,3,9$ & 1,0 & 2,1 & 3,1 & $1,1,1$ & $0,0,0$ & $1,1,1$ \\
$(1,0,0)$ & $3,2,2$ & $2,1,1$ & $1,1,1$ & $1,1,1$ & $7,5,5$ & 3,2 & 2,1 & 5,3 & $1,1,1$ & $1,1,2$ & $2,2,3$ \\
$(1,0,1)$ & $2,1,1$ & $1,0,0$ & $1,0,0$ & $0,0,0$ & $4,1,1$ & 2,1 & 1,0 & 3,1 & $1,2,2$ & $0,0,1$ & $1,2,3$ \\
$(1,1,0)$ & $2,1,3$ & $1,2,2$ & $1,1,2$ & $1,1,2$ & $5,5,9$ & 2,1 & 1,0 & 3,1 & $1,2,2$ & $1,1,1$ & $2,3,3$ \\
\hline
\end{tabular}

The interpretation $(a, b, c)=(0,1,0)$ is a model of ${ }^{*} \Delta_{\mu}^{d_{H}, \Sigma}\left(E_{1}\right) \wedge{ }^{*} \Delta_{\mu}^{d_{H}, \Sigma}\left(E_{2}\right)$, but not of ${ }^{*} \Delta_{\mu}^{d_{H}, \Sigma}\left(E_{1} \sqcup E_{2}\right)$.

(IC6) The example above is a counter-example to (IC6), since the interpretation $(a, b, c)=(1,0,1)$ is a model of ${ }^{*} \Delta_{\mu}^{d_{H}, \Sigma}\left(E_{1} \sqcup E_{2}\right)$ but not of ${ }^{*} \Delta_{\mu}^{d_{H}, \Sigma}\left(E_{1}\right) \wedge{ }^{*} \Delta_{\mu}^{d_{H}, \Sigma}\left(E_{2}\right)$.

(Maj) Consider two given profiles $E_{1}$ and $E_{2}$. For a given $\omega$, we have $\forall i, d_{\Sigma}$ $(\omega, E_{1}^{i} \sqcup \underbrace{E_{2}^{i} \sqcup \ldots \sqcup E_{2}^{i}})=d_{\Sigma}\left(\omega, E_{1}^{i}\right)+m \cdot d_{\Sigma}\left(\omega, E_{2}^{i}\right)$. So for a sufficiently large $m$ (for instance by ${ }^{m}$ choosing an $\left.m>\max _{\omega, \omega^{\prime} \in \mathcal{W}} d\left(\omega, \omega^{\prime}\right)\right)$, we have $\forall i, \Delta_{\mu}\left(E_{1}^{i} \sqcup\right.$ $\underbrace{E_{2}^{i} \sqcup \ldots \sqcup E_{2}^{i}}) \equiv \Delta_{\mu}\left(E_{2}^{i}\right)$. By choosing a sufficiently large $i$ (such that the fixed points $^{m}$ of $\left(\Delta_{\mu}(E_{1}^{i} \sqcup \underbrace{E_{2}^{i} \sqcup \ldots \sqcup E_{2}^{i}}_{m})\right)_{i \geq 0}$ and of $\left(\Delta_{\mu}\left(E_{2}^{i}\right)\right)_{i \geq 0}$ are reached), we get the
postulate (Maj).

(Arb) The following table shows a counter-example to the (Arb) postulate. We consider three propositional symbols and the profile $E=\left(K_{1}, K_{2}\right)$.

\begin{tabular}{l|cc|cc|c||c||c}
\hline $\mathcal{W}$ & $\mu_{1}$ & $\mu_{2}$ & $K_{1}^{i}$ & $K_{2}^{i}$ & $E^{i}$ & ${ }^{*} \Delta_{\mu_{1}}^{d_{H}, \Sigma}\left(K_{1}\right)$ & ${ }^{*} \Delta_{\mu_{2}}^{d_{H}, \Sigma}\left(K_{2}\right)$ \\
\hline$(0,0,0)$ & 0 & 0 & 1 & 0 & 1 & 0 & 0 \\
$(0,0,1)$ & 0 & 0 & 2 & 1 & 3 & 1 & 1 \\
$(0,1,0)$ & 1 & 0 & 0 & 1 & 1 & 1 & 1 \\
$(0,1,1)$ & 1 & 1 & 1 & 2 & 3 & 2 & 2 \\
$(1,0,0)$ & 0 & 1 & 2 & 1 & 3 & 1 & 1 \\
$(1,0,1)$ & 0 & 0 & 3 & 2 & 5 & 2 & 2 \\
$(1,1,0)$ & 0 & 0 & 1 & 0 & 1 & 0 & 0 \\
$(1,1,1)$ & 1 & 1 & 2 & 1 & 3 & 1 & 1 \\
\hline
\end{tabular}

For the sceptical CHIM operator $\triangle_{\mu}^{* d_{H}, \Sigma}$, the proofs are:

(IC0-IC3), (IC7), (IC8) see Proposition 12.

(IC5) The following table shows a counter-example to (IC5). We consider a language with two propositional symbols and the profiles $E_{1}=\left(K_{1}, K_{2}\right), E_{2}=\left(K_{3}, K_{4}\right)$ and $E=E_{1} \sqcup E_{2}$. 
Conciliation through Iterated Belief Merging 933

\begin{tabular}{l|llll|l||ll|l||ll|l}
\hline $\mathcal{W}$ & $K_{1}^{i}$ & $K_{2}^{i}$ & $K_{3}^{i}$ & $K_{4}^{i}$ & $E_{\Delta_{\mu}\left(E^{i}\right)}^{i}$ & $K_{1}^{i}$ & $K_{2}^{i}$ & $E_{1 \Delta_{\mu}\left(E_{1}^{i}\right)}^{i}$ & $K_{3}^{i}$ & $K_{4}^{i}$ & $E_{2 \Delta_{\mu}\left(E_{2}^{i}\right)}^{i}$ \\
\hline$(0,0)$ & 1,1 & 1,2 & 0,1 & 0,1 & $2_{1}, 5_{1}$ & 1,1 & 1,1 & $2_{1}, 2_{0}$ & 0 & 0 & $0_{0}$ \\
$(0,1)$ & 0,0 & 1,1 & 0,0 & 0,0 & $1_{0}, 1_{0}$ & 0,0 & 1,2 & $1_{0}, 2_{0}$ & 0 & 0 & $0_{0}$ \\
$(1,0)$ & 1,2 & 0,1 & 1,2 & 1,2 & $3_{2}, 7_{2}$ & 1,2 & 0,0 & $1_{0}, 2_{0}$ & 1 & 1 & $2_{1}$ \\
\hline
\end{tabular}

So, in this case $\Delta_{\mu}^{* d_{H}, \Sigma}\left(E_{1}\right) \wedge \Delta_{\mu}^{* d_{H}, \Sigma}\left(E_{2}\right) \not \forall \Delta_{\mu}^{* d_{H}, \Sigma}\left(E_{1} \sqcup E_{2}\right)$.

(IC6) The following table gives a counter-example to (IC6). We consider a language with two propositional symbols and the profiles $E_{1}=\left(K_{1}, K_{2}\right), E_{2}=\left(K_{3}, K_{4}\right)$ and $E=E_{1} \sqcup E_{2}$.

\begin{tabular}{l|llll|l||ll|l||ll|l}
\hline $\mathcal{W}$ & $K_{1}^{i}$ & $K_{2}^{i}$ & $K_{3}^{i}$ & $K_{4}^{i}$ & $E_{\Delta_{\mu}\left(E^{i}\right)}^{i}$ & $K_{1}^{i}$ & $K_{2}^{i}$ & $E_{1 \Delta_{\mu}\left(E_{1}^{i}\right)}^{i}$ & $K_{3}^{i}$ & $K_{4}^{i}$ & $E_{2 \Delta_{\mu}\left(E_{2}^{i}\right)}^{i}$ \\
\hline$(0,0)$ & 0 & 1 & 1 & 0 & $2_{0}$ & 0 & 1 & $1_{0}$ & $1,1,1$ & $0,0,0$ & $1_{0}, 1_{0}, 1_{0}$ \\
$(0,1)$ & 1 & 0 & 1 & 0 & $2_{0}$ & 1 & 0 & $1_{0}$ & $1,2,2$ & $0,0,1$ & $1_{0}, 2_{1}, 3_{1}$ \\
$(1,0)$ & 1 & 2 & 0 & 1 & $4_{1}$ & 1 & 2 & $3_{1}$ & $0,0,0$ & $1,1,1$ & $1_{0}, 1_{0}, 1_{0}$ \\
\hline
\end{tabular}

Here $\Delta_{\mu}^{* d_{H}, \Sigma}\left(E_{1}\right) \wedge \Delta_{\mu}^{* d_{H}, \Sigma}\left(E_{2}\right)$ is consistent, and $\Delta_{\mu}^{* d_{H}, \Sigma}\left(E_{1} \sqcup E_{2}\right) \not \forall \Delta_{\mu}^{* d_{H}, \Sigma}\left(E_{1}\right) \wedge$ $\Delta_{\mu}^{* d_{H}, \Sigma}\left(E_{2}\right)$.

(Maj) Consider two given profiles $E_{1}$ and $E_{2}$. For a given $\omega$ we have $\forall i, d(\omega, E_{1}^{i} \sqcup \underbrace{E_{2}^{i} \sqcup \ldots \sqcup E_{2}^{i}}_{m})=d\left(\omega, E_{1}^{i}\right)+\operatorname{m.d}\left(\omega, E_{2}^{i}\right)$. So for a sufficiently large $m$ (for instance by choosing $\left.m>\max _{\omega, \omega^{\prime} \in \mathcal{W}} d\left(\omega, \omega^{\prime}\right)\right)$, we get $\forall i, \Delta_{\mu}(E_{1}^{i} \sqcup \underbrace{E_{2}^{i} \sqcup \ldots \sqcup E_{2}^{i}}_{m}) \equiv$ $\Delta_{\mu}\left(E_{2}^{i}\right)$. By choosing a sufficiently large $i$ (such that the fixed points of $\left(\Delta_{\mu}(E_{1}^{i} \sqcup \underbrace{\left.E_{2}^{i} \sqcup \ldots \sqcup E_{2}^{i}\right)}_{m})_{i \geq 0}\right.$ and of $\left(\Delta_{\mu}\left(E_{2}^{i}\right)\right)_{i \geq 0}$ are reached), we get (Maj).

(Arb) The following table gives a counter-example to (Arb). We consider a language with three propositional symbols and the profile $E=\left(K_{1}, K_{2}\right)$.

\begin{tabular}{l|ll|ll|l||l||l}
\hline $\mathcal{W}$ & $\mu_{1}$ & $\mu_{2}$ & $K_{1}$ & $K_{2}$ & $E_{\Delta_{\mu_{1} \vee d_{2}}^{*}, \Sigma}$ & $\Delta_{\mu_{1}}^{* d_{H}, \Sigma}\left(K_{1}\right)$ & $\Delta_{\mu_{2}}^{* d_{H}, \Sigma}\left(K_{2}\right)$ \\
\hline$(0,0,0)$ & 0 & 0 & 1 & 0 & $1_{0}$ & 0 & 0 \\
$(0,0,1)$ & 0 & 0 & 2 & 1 & $3_{1}$ & 1 & 1 \\
$(0,1,0)$ & 1 & 0 & 0 & 1 & $1_{0}$ & 1 & 1 \\
$(0,1,1)$ & 1 & 1 & 1 & 2 & $3_{1}$ & 1 & 2 \\
$(1,0,0)$ & 0 & 1 & 2 & 1 & $3_{1}$ & 2 & 1 \\
$(1,0,1)$ & 0 & 0 & 3 & 2 & $5_{2}$ & 0 & 2 \\
$(1,1,0)$ & 0 & 0 & 1 & 0 & $1_{0}$ & 1 & 0 \\
$(1,1,1)$ & 1 & 1 & 2 & 1 & $3_{1}$ & 1 & 1 \\
\hline
\end{tabular}

Proof of Proposition 16. For the credulous CHIM operator ${ }^{*} \triangle_{\mu}^{d_{H}, \text { Max }}$ the proofs are:

(IC0-IC3), (IC7), (IC8) see Proposition 12.

(IC5) The following table gives a counter-example to (IC5). We consider a language with two propositional symbols, and we consider the profiles $E_{1}=\left(K_{1}, K_{2}\right), E_{2}=\left(K_{3}, K_{4}\right)$ and $E=E_{1} \sqcup E_{2}$. 
934 Conciliation through Iterated Belief Merging

\begin{tabular}{l|llll|l||ll|l||ll|l}
\hline $\mathcal{W}$ & $K_{1}^{i}$ & $K_{2}^{i}$ & $K_{3}^{i}$ & $K_{4}^{i}$ & $E^{i}$ & $K_{1}^{i}$ & $K_{2}^{i}$ & $E_{1}^{i}$ & $K_{3}^{i}$ & $K_{4}^{i}$ & $E_{2}^{i}$ \\
\hline$(0,0)$ & $0,1,0$ & $2,1,0$ & $1,1,0$ & $0,1,0$ & $2,1,0$ & 0,1 & 2,1 & 2,1 & $1,1,1$ & $0,0,0$ & $1,1,1$ \\
$(0,1)$ & $1,0,1$ & $1,0,1$ & $0,0,1$ & $1,2,1$ & $1,2,1$ & 1,0 & 1,0 & 1,0 & $0,0,0$ & $1,1,1$ & $1,1,1$ \\
$(1,0)$ & $1,0,1$ & $1,0,1$ & $1,2,1$ & $0,0,1$ & $1,2,1$ & 1,0 & 1,0 & 1,0 & $1,2,2$ & $0,0,1$ & $1,2,2$ \\
\hline
\end{tabular}

The interpretation $(0,1)$ is a model of ${ }^{*} \Delta_{\mu}^{d_{H}, \operatorname{Max}}\left(E_{1}\right) \wedge{ }^{*} \Delta_{\mu}^{d_{H}, \operatorname{Max}}\left(E_{2}\right)$, but not of ${ }^{*} \Delta_{\mu}^{d_{H}, \operatorname{Max}}\left(E_{1} \sqcup E_{2}\right)$.

(IC6) The example above is a counter-example to (IC6), since the interpretation $(0,0)$ is a model of ${ }^{*} \Delta_{\mu}^{d_{H}, \operatorname{Max}}\left(E_{1} \sqcup E_{2}\right)$ but not of ${ }^{*} \Delta_{\mu}^{d_{H}, \operatorname{Max}}\left(E_{1}\right) \wedge{ }^{*} \Delta_{\mu}^{d_{H}, \operatorname{Max}}\left(E_{2}\right)$.

(Maj) Let us consider the following counter-example. Let us consider a language with two propositional symbols $a$ and $b$, and two bases $K_{1} \equiv \neg a \wedge \neg b$ and $K_{2} \equiv \neg a \wedge b$, with $\mu \equiv \top$, we get $\quad{ }^{*} \Delta_{\mu}^{d_{H}, \operatorname{Max}}(E_{1}^{i} \sqcup \underbrace{E_{2}^{i} \sqcup \ldots \sqcup E_{2}^{i}}_{m}) \equiv$
${ }^{*} \Delta_{\mu}^{d_{H}, \operatorname{Max}}\left(E_{1} \sqcup E_{2}\right) \equiv \neg a$, and ${ }^{*} \Delta_{\mu}^{d_{H}, \operatorname{Max}}\left(E_{2}\right) \equiv \neg a \wedge b$.

(Arb) The following table provides a counter-example to (Arb). We consider a language with three propositional symbols and the profile $E=\left(K_{1}, K_{2}\right)$.

\begin{tabular}{l|cc|cc|c||c||c}
\hline $\mathcal{W}$ & $\mu_{1}$ & $\mu_{2}$ & $K_{1}^{i}$ & $K_{2}^{i}$ & $E^{i}$ & $* \Delta_{\mu_{1}}^{d_{H}, \operatorname{Max}}\left(K_{1}\right)$ & $* \Delta_{\mu_{2}}^{d_{H}, \operatorname{Max}}\left(K_{2}\right)$ \\
\hline$(0,0,0)$ & 0 & 0 & 1 & 0 & 1 & 0 & 0 \\
$(0,0,1)$ & 0 & 0 & 2 & 1 & 2 & 1 & 1 \\
$(0,1,0)$ & 1 & 0 & 0 & 1 & 1 & 1 & 1 \\
$(0,1,1)$ & 1 & 1 & 1 & 2 & 2 & 2 & 2 \\
$(1,0,0)$ & 0 & 1 & 2 & 1 & 2 & 1 & 1 \\
$(1,0,1)$ & 0 & 0 & 3 & 2 & 3 & 2 & 0 \\
$(1,1,0)$ & 0 & 0 & 1 & 0 & 1 & 0 & 1 \\
$(1,1,1)$ & 1 & 1 & 2 & 1 & 2 & 1 & 0 \\
\hline
\end{tabular}

For the credulous CHIM operator ${ }^{*} \triangle_{\mu}^{d_{H}, \text { GMax }}$ the proofs are:

(IC0-IC3), (IC7), (IC8) see Proposition 12.

(IC5) The following table gives a counter-example to (IC5). We consider a language with two propositional symbols $\{a, b\}$, and the profiles $E_{1}=\left(K_{1}, K_{2}\right), E_{2}=\left(K_{3}, K_{4}\right)$ and $E=E_{1} \sqcup E_{2}$.

\begin{tabular}{|c|c|c|c|c|c|c|}
\hline $\mathcal{W}$ & $K_{1}^{i}$ & $K_{2}^{i}$ & $K_{3}^{i}$ & $K_{4}^{i}$ & \multicolumn{2}{|l|}{$E^{i}$} \\
\hline$(0,0)$ & $0,1,0$ & $2,1,0$ & $1,1,0$ & $0,1,0$ & \multicolumn{2}{|c|}{$(2,1,0,0),(1,1,1,1),(0,0,0,0$} \\
\hline$(0,1)$ & $1,0,1$ & $1,0,1$ & $0,0,1$ & 1 , & \multicolumn{2}{|c|}{$(1,1,1,0),(2,0,0,0),(1,1,1,1$} \\
\hline$(1,0)$ & $1,0,1$ & $1,0,1$ & $1,2,1$ & $0,0,1$ & \multicolumn{2}{|c|}{$(1,1,1,0),(2,0,0,0),(1,1,1,1$} \\
\hline $\mathcal{W}$ & $K_{1}^{i}$ & $K_{2}^{i}$ & $E_{1}^{i}$ & $K_{3}^{i}$ & $K_{4}^{i}$ & $E_{2}^{i}$ \\
\hline & 0,1 & 2,1 & $0),(1,1)$ & $1,1,1$ & $0,0,0$ & $(1,0)$, \\
\hline 0 & 1, & 1,0 & ),$(0$ & 0 , & & $(1,0),(1,0)$ \\
\hline$(1,0)$ & 1,0 & 1,0 & $(1,1),(0,0)$ & $1,2,2$ & $0,0,1$ & $(1,0),(2,0),(2,1$ \\
\hline
\end{tabular}


The interpretation $(0,1)$ is a model of ${ }^{*} \Delta_{\mu}^{d_{H}, \operatorname{GMax}}\left(E_{1}\right) \wedge{ }^{*} \Delta_{\mu}^{d_{H}, \operatorname{GMax}}\left(E_{2}\right)$, but not of ${ }^{*} \Delta_{\mu}^{d_{H}, \operatorname{GMax}}\left(E_{1} \sqcup E_{2}\right)$.

(IC6) The example above is also a counter-example to (IC6), since the interpretation $(0,0)$ is a model of ${ }^{*} \Delta_{\mu}^{d_{H}, \operatorname{GMax}}\left(E_{1} \sqcup E_{2}\right)$ but not of ${ }^{*} \Delta_{\mu}^{d_{H}, \operatorname{GMax}}\left(E_{1}\right) \wedge{ }^{*} \Delta_{\mu}^{d_{H}, \operatorname{GMax}}\left(E_{2}\right)$.

(Maj) The following table gives a counter-example to (Maj). We consider a language with two propositional symbols and the profile $E=(K_{1}, \underbrace{K_{2}, \ldots, K_{2}}_{m})$.

\begin{tabular}{l|llll|l||l|l}
\hline $\mathcal{W}$ & $K_{1}^{i}$ & $K_{2}^{i}$ & $\ldots$ & $K_{2}^{i}$ & $E_{\Delta_{\mu}\left(E^{i}\right)}^{i}$ & $K_{2}^{i}$ & $K_{2 \Delta_{\mu}\left(K_{2}^{i}\right)}^{i}$ \\
\hline$(0,0)$ & 2,2 & 0,2 & $\ldots$ & 0,2 & $(2,0, \ldots, 0)_{2},(2,2, \ldots, 2)_{2}$ & 0,0 & $0_{0}, 0_{0}$ \\
$(0,1)$ & 1,1 & 1,1 & $\ldots$ & 1,1 & $(1,1, \ldots, 1)_{1},(1,1, \ldots, 1)_{1}$ & 1,1 & $1_{1}, 1_{1}$ \\
$(1,1)$ & 0,0 & 1,0 & $\ldots$ & 1,0 & $(1, \ldots, 1,0)_{0},(0,0, \ldots, 0)_{0}$ & 1,2 & $1_{2}, 2_{2}$ \\
\hline
\end{tabular}

So we have $\forall m,{ }^{*} \Delta_{\mu}^{d_{H}, \operatorname{GMax}}(E_{1}^{i} \sqcup \underbrace{E_{2}^{i} \sqcup \ldots \sqcup E_{2}^{i}}_{m}) \not{ }^{*} \Delta_{\mu}^{d_{H}, \operatorname{GMax}}\left(E_{2}\right)$.

(Arb) The following table shows a counter-example to (Arb). We consider a language with three propositional symbols, and the profile $E=\left(K_{1}, K_{2}\right)$.

\begin{tabular}{l|cc|cc|c||c||c}
\hline $\mathcal{W}$ & $\mu_{1}$ & $\mu_{2}$ & $K_{1}^{i}$ & $K_{2}^{i}$ & $E^{i}$ & ${ }^{*} \Delta_{\mu_{1}}^{d_{H}, \mathrm{GMax}}\left(K_{1}\right)$ & ${ }^{*} \Delta_{\mu_{2}}^{d_{H}, \mathrm{GMax}}\left(K_{2}\right)$ \\
\hline$(0,0,0)$ & 0 & 0 & 1 & 0 & $(1,0)$ & 0 & 0 \\
$(0,0,1)$ & 0 & 0 & 2 & 1 & $(2,1)$ & 1 & 1 \\
$(0,1,0)$ & 1 & 0 & 0 & 1 & $(1,0)$ & 1 & 1 \\
$(0,1,1)$ & 1 & 1 & 1 & 2 & $(2,1)$ & 2 & 2 \\
$(1,0,0)$ & 0 & 1 & 2 & 1 & $(2,1)$ & 1 & 1 \\
$(1,0,1)$ & 0 & 0 & 3 & 2 & $(3,2)$ & 2 & 2 \\
$(1,1,0)$ & 0 & 0 & 1 & 0 & $(1,0)$ & 0 & 0 \\
$(1,1,1)$ & 1 & 1 & 2 & 1 & $(2,1)$ & 1 & 1 \\
\hline
\end{tabular}

For the sceptical CHIM operator $\triangle_{\mu_{1}}^{* d_{H}, \text { Max }}$ the proofs are:

(IC0-IC3), (IC7), (IC8) see Proposition 12.

(IC5) The following table gives a counter-example to (IC5). Consider a language with three propositional symbols and the profiles $E_{1}=\left(K_{1}, K_{2}\right), E_{2}=\left(K_{3}, K_{4}\right)$ and $E=E_{1} \sqcup E_{2}$.

\begin{tabular}{l|cccc|c||c||c}
\hline $\mathcal{W}$ & $K_{1}^{i}$ & $K_{2}^{i}$ & $K_{3}^{i}$ & $K_{4}^{i}$ & $E_{\Delta_{\mu}\left(E^{i}\right)}^{i}$ & $E_{1 \Delta_{\mu}\left(E_{1}^{i}\right)}^{i}$ & $E_{2 \Delta_{\mu}\left(E_{2}^{i}\right)}^{i}$ \\
\hline$(0,0,0)$ & 0,0 & 3,3 & 1,1 & 1,1 & $3_{1}, 3_{1}$ & $3_{1}$ & $1_{0}$ \\
$(0,0,1)$ & 1,1 & 2,2 & 0,0 & 2,2 & $2_{0}, 2_{0}$ & $2_{0}$ & $2_{1}$ \\
$(0,1,0)$ & 1,1 & 2,2 & 2,2 & 0,0 & $2_{0}, 2_{0}$ & $2_{0}$ & $2_{1}$ \\
$(1,0,0)$ & 1,1 & 2,2 & 2,2 & 2,2 & $2_{0}, 2_{0}$ & $2_{0}$ & $2_{1}$ \\
$(1,0,1)$ & 2,2 & 1,1 & 1,1 & 1,3 & $2_{0}, 3_{1}$ & $2_{0}$ & $1_{0}$ \\
$(1,1,0)$ & 2,2 & 1,1 & 3,3 & 1,1 & $3_{1}, 3_{1}$ & $2_{0}$ & $3_{2}$ \\
\hline
\end{tabular}

One can check that $\Delta_{\mu}^{* d_{H}, \operatorname{Max}}\left(E_{1}\right) \wedge \Delta_{\mu}^{* d_{H}}, \operatorname{Max}\left(E_{2}\right) \not \forall \Delta_{\mu}^{* d_{H}, \operatorname{Max}}\left(E_{1} \sqcup E_{2}\right)$.

(IC6) The following table gives a counter-example to (IC6). Consider a language with three propositional symbols and consider the profiles $E_{1}=\left(K_{1}\right), E_{2}=\left(K_{2}\right)$. 


\begin{tabular}{l|cc|c||c||c}
\hline $\mathcal{W}$ & $K_{1}^{i}$ & $K_{2}^{i}$ & $E_{\Delta_{\mu}\left(E^{i}\right)}^{i}$ & $\Delta_{\mu}\left(K_{1}^{i}\right)$ & $\Delta_{\mu}\left(K_{2}^{i}\right)$ \\
\hline$(0,0,0)$ & 2,2 & 0,1 & $2_{1}, 2_{1}$ & 1 & 0 \\
$(0,0,1)$ & 1,1 & 0,0 & $1_{0}, 1_{0}$ & 0 & 0 \\
$(0,1,0)$ & 1,1 & 1,2 & $1_{0}, 2_{2}$ & 0 & 1 \\
$(1,0,0)$ & 2,2 & 1,2 & $2_{1}, 2_{1}$ & 1 & 1 \\
$(1,0,1)$ & 1,1 & 1,1 & $1_{0}, 1_{0}$ & 0 & 1 \\
$(1,1,0)$ & 1,1 & 2,3 & $2_{1}, 3_{2}$ & 0 & 2 \\
\hline
\end{tabular}

(Maj) We have that $\forall m, \Delta_{\mu}^{d_{H}, \operatorname{Max}}(E_{1}^{i} \sqcup \underbrace{E_{2}^{i} \sqcup \ldots \sqcup E_{2}^{i}}_{m}) \equiv \Delta_{\mu}\left(E_{1} \sqcup E_{2}\right)$. Straightforwardly, this property remains true at each iteration, so $\forall m, \Delta_{\mu}^{*} d_{H}, \operatorname{Max}(E_{1}^{i} \sqcup \underbrace{E_{2}^{i} \sqcup \ldots \sqcup E_{2}^{i}}_{2}) \equiv$ $\Delta_{\mu}^{* d_{H}, \operatorname{Max}}\left(E_{1} \sqcup E_{2}\right)$. The above table that gives a counter-example to (IC6) shows a case where $\Delta_{\mu}^{* d_{H}, \operatorname{Max}}\left(E_{1} \sqcup E_{2}\right) \not \forall \Delta_{\mu}^{* d_{H}, \operatorname{Max}}\left(E_{2}\right)$.

(Arb) The following table gives a counter-example to (Arb). Consider a language with three propositional symbols, and consider the profile $E=\left(K_{1}, K_{2}\right)$.

\begin{tabular}{l|cc|cc|c||c||c}
\hline $\mathcal{W}$ & $\mu_{1}$ & $\mu_{2}$ & $K_{1}$ & $K_{2}$ & $E_{\Delta_{\mu_{1} d_{2}}^{* d_{H}, \operatorname{ax}}}(E)$ & $\Delta_{\mu_{1}}^{* d_{H}, \operatorname{Max}}\left(K_{1}\right)$ & $\Delta_{\mu_{2}}^{* d_{H}, \operatorname{Max}}\left(K_{2}\right)$ \\
\hline$(0,0,0)$ & 0 & 0 & 1 & 0 & $1_{0}$ & 0 & 0 \\
$(0,0,1)$ & 0 & 0 & 2 & 1 & $2_{1}$ & 1 & 1 \\
$(0,1,0)$ & 1 & 0 & 0 & 1 & $1_{0}$ & 1 & 1 \\
$(0,1,1)$ & 1 & 1 & 1 & 2 & $2_{1}$ & 2 & 2 \\
$(1,0,0)$ & 0 & 1 & 2 & 1 & $2_{1}$ & 1 & 1 \\
$(1,0,1)$ & 0 & 0 & 3 & 2 & $3_{2}$ & 2 & 2 \\
$(1,1,0)$ & 0 & 0 & 1 & 0 & $1_{0}$ & 0 & 0 \\
$(1,1,1)$ & 1 & 1 & 2 & 1 & $2_{1}$ & 1 & 1 \\
\hline
\end{tabular}

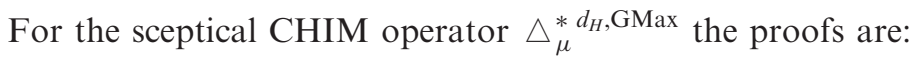

(IC0-IC3), (IC7), (IC8) see Proposition 12.

(IC5) The following tables give a counter-example to (IC5). Consider a language with two propositional symbols, and consider the profiles $E_{1}=\left(K_{1}, K_{2}\right), E_{2}=\left(K_{3}, K_{4}\right)$ and $E=E_{1} \sqcup E_{2}$.

We get $\Delta_{\mu}^{* d_{H}, \operatorname{GMax}}\left(E_{1}\right) \wedge \Delta_{\mu}^{* d_{H}, \operatorname{GMax}}\left(E_{2}\right) \not \forall \Delta_{\mu}^{* d_{H}, \operatorname{GMax}}\left(E_{1} \sqcup E_{2}\right)$.

\begin{tabular}{l|llll|l}
\hline $\mathcal{W}$ & $K_{1}^{i}$ & $K_{2}^{i}$ & $K_{3}^{i}$ & $K_{4}^{i}$ & $E_{\Delta_{\mu}\left(E^{i}\right)}^{i}$ \\
\hline$(0,0)$ & 1,1 & 1,1 & 1,1 & 0,1 & $(1,1,1,0)_{1},(1,1,1,1)_{0}$ \\
$(0,1)$ & 0,0 & 0,0 & 0,0 & 1,2 & $(1,0,0,0)_{0},(2,0,0,0)_{1}$ \\
$(1,0)$ & 0,0 & 0,0 & 1,2 & 0,0 & $(1,0,0,0)_{0},(2,0,0,0)_{1}$ \\
\hline
\end{tabular}

\begin{tabular}{l|ll|l||ll|l}
\hline $\mathcal{W}$ & $K_{1}^{i}$ & $K_{2}^{i}$ & $E_{1 \Delta_{\mu}\left(E_{1}^{i}\right)}^{i}$ & $K_{3}^{i}$ & $K_{4}^{i}$ & $E_{2_{\Delta_{\mu}}\left(E_{2}^{i}\right)}^{i}$ \\
\hline$(0,0)$ & 1 & 1 & $(1,1)_{1}$ & $1,1,1$ & $0,0,0$ & $(1,0)_{0},(1,0)_{0},(1,0)_{0}$ \\
$(0,1)$ & 0 & 0 & $(0,0)_{0}$ & $0,0,0$ & $1,1,1$ & $(1,0)_{0},(1,0)_{0},(1,0)_{0}$ \\
$(1,0)$ & 0 & 0 & $(0,0)_{0}$ & $1,2,2$ & $0,0,1$ & $(1,0)_{0},(2,0)_{1},(2,1)_{1}$ \\
\hline
\end{tabular}


(IC6) The example above is also a counter-example to (IC6), since $\Delta_{\mu}^{* d_{H}, \mathrm{GMax}}\left(E_{1}\right) \wedge$ $\Delta_{\mu}^{* d_{H}, \operatorname{GMax}}\left(E_{2}\right) \quad$ is consistent, and $\Delta_{\mu}^{* d_{H}, \operatorname{GMax}}\left(E_{1} \sqcup E_{2}\right) \not \Delta_{\mu}^{* d_{H}, \operatorname{GMax}}\left(E_{1}\right) \wedge$ $\Delta_{\mu}^{*} d_{H}, \operatorname{GMax}\left(E_{2}\right)$.

(Maj) The following table gives a counter-example to (Maj). Consider a language with two propositional symbols, and consider the profile $E=(K_{1}, \underbrace{K_{2}, \ldots, K_{2}}_{m})$.

\begin{tabular}{l|ll|l||l|l}
\hline $\mathcal{W}$ & $K_{1}^{i}$ & $K_{2}^{i}$ & $E_{\Delta_{\mu}\left(E^{i}\right)}^{i}$ & $K_{2}^{i}$ & $\left(K_{2}^{i}\right)_{\Delta_{\mu}\left(K_{2}^{i}\right)}$ \\
\hline$(0,0)$ & 2,2 & 0,1 & $(2,0, \ldots, 0)_{2},(2,1, \ldots, 1)_{2}$ & 0,0 & $0_{0}, 0_{0}$ \\
$(0,1)$ & 1,1 & 1,2 & $(1,1, \ldots, 1)_{1},(2, \ldots, 2,1)_{1}$ & 1,1 & $1_{1}, 1_{1}$ \\
$(1,1)$ & 0,0 & 1,1 & $(1, \ldots, 1,0)_{0},(1, \ldots, 1,0)_{0}$ & 1,2 & $1_{2}, 2_{2}$ \\
\hline
\end{tabular}

We have $\forall m, \Delta_{\mu}^{* d_{H}, \operatorname{GMax}}(E_{1} \sqcup \underbrace{E_{2} \sqcup \ldots \sqcup E_{2}}_{m}) \not \forall \Delta_{\mu}^{* d_{H}, \operatorname{GMax}}\left(E_{2}\right)$.

(Arb) The following table is a counter-example to (Arb). Consider a language with three propositional symbols, and consider the profile $E=\left(K_{1}, K_{2}\right)$.

\begin{tabular}{l|cc|cc|c||c||c}
\hline $\mathcal{W}$ & $\mu_{1}$ & $\mu_{2}$ & $K_{1}$ & $K_{2}$ & $E_{\Delta_{\mu_{1} \vee \mu_{2}}^{*} d_{H, G a x}}$ & $\Delta_{\mu_{1}}^{* d_{H}, \mathrm{GMax}}\left(K_{1}\right)$ & $\Delta_{\mu_{2}}^{* d_{H}, \mathrm{GMax}}\left(K_{2}\right)$ \\
\hline$(0,0,0)$ & 0 & 0 & 1 & 0 & $(1,0)_{0}$ & 0 & 0 \\
$(0,0,1)$ & 0 & 0 & 2 & 1 & $(2,1)_{1}$ & 1 & 1 \\
$(0,1,0)$ & 1 & 0 & 0 & 1 & $(1,0)_{0}$ & 1 & 1 \\
$(0,1,1)$ & 1 & 1 & 1 & 2 & $(2,1)_{1}$ & 2 & 2 \\
$(1,0,0)$ & 0 & 1 & 2 & 1 & $(2,1)_{1}$ & 1 & 1 \\
$(1,0,1)$ & 0 & 0 & 3 & 2 & $(3,2)_{2}$ & 2 & 2 \\
$(1,1,0)$ & 0 & 0 & 1 & 0 & $(1,0)_{0}$ & 0 & 0 \\
$(1,1,1)$ & 1 & 1 & 2 & 1 & $(2,1)_{1}$ & 1 & 1 \\
\hline
\end{tabular}

